\title{
Thermal-viscous instability in tilted accretion disks: a possible application to IW And-type dwarf novae
}

\author{
Mariko KImUra $^{1, *}$, Yoji OSAKI ${ }^{2}$, Taichi Kato ${ }^{1}$, and Shin Mineshige ${ }^{1}$ \\ ${ }^{1}$ Department of Astronomy, Graduate School of Science, Kyoto University, Oiwakecho, \\ Kitashirakawa, Sakyo-ku, Kyoto 606-8502 \\ ${ }^{2}$ Department of Astronomy, School of Science, University of Tokyo, Hongo, Tokyo 113-0033 \\ *E-mail: mkimura@kusastro.kyoto-u.ac.jp \\ Received; Accepted
}

\begin{abstract}
IW And stars are a subgroup of dwarf novae characterized by repetitive light variations of the intermediate-brightness state with oscillations, which is terminated by brightening. This group of dwarf novae is also known to exhibit a wide variety even within one system in long-term light curves including usual dwarf-nova outbursts, Z Cam-type standstills, and so on, besides the typical IW And-type variations mentioned above. Following the recent observations suggesting that some IW And stars seem to have tilted disks, we have investigated how the thermalviscous instability works in tilted accretion disks in dwarf novae and whether it could reproduce the essential features of the light curves in IW And stars. By adopting various simplifying assumptions for tilted disks, we have performed time-dependent one-dimensional numerical simulations of a viscous disk by taking into account various mass supply patterns to the disk; that is, the gas stream from the secondary star flows not only to the outer edge of the disk but also to the inner portions of the disk. We find that tilted disks can achieve a new kind of accretion cycle, in which the inner disk almost always stays in the hot state while the outer disk repeats outbursts, thereby reproducing alternating mid-brightness interval sometimes with dips and brightening, which are quite reminiscent of the most characteristic observational light variations of IW And stars. Further, we have found that our simulations produce diverse light variations, depending on different mass supply patterns even without time variations in mass transfer rates. This could explain the wide variety in long-term light curves of IW And stars.
\end{abstract}

Key words: accretion, accretion disks - instabilities - binaries: close - novae, cataclysmic variables stars: dwarf novae

\section{Introduction}

Cataclysmic Variables (CVs) are close binary systems composed of a white dwarf (the primary) and a low-mass cool star filling the critical Roche lobe (the secondary). An accretion disk is formed around the primary white dwarf via Roche-lobe overflow from the secondary in non-magnetic CVs. Dwarf novae (DNe), one subclass of CVs, exhibit transient events called "outbursts" with amplitudes of 2-6 mag with a typical timescale of several 10 days due to sudden brightening of accretion disks (Warner 1995 for a general review).

Thermal limit-cycle instability explains normal dwarf-nova outbursts (Osaki 1996 for a review). Partial ionization of hydrogen triggers thermal instability in a disk (Hōshi 1979), and the disk shows bi-stable states: the hot state with a high accretion rate and the cool state with a low accretion rate. The disk jumps between the two stable states, because they sandwich an unsta-

(C) 2014. Astronomical Society of Japan. 
ble state on the thermal equilibrium curve, which appears due to significant effect of convection (Meyer, Meyer-Hofmeister 1981). This limit cycle drastically increases/decreases the mass accretion rate onto the primary white dwarf. If the mass transfer rate from the secondary star is less than the minimum rate for keeping the entire disk hot, which is denoted as $\dot{M}_{\text {crit }}$, the systems experience sporadic outbursts. If the mass transfer rate is higher than $\dot{M}_{\text {crit }}$, the systems show constant high disk luminosity, and are called nova-like stars (NLs) (Smak 1983).

However, we cannot understand several kinds of dwarf-nova outbursts only by the simple disk instability model with constant mass transfer rates from the secondary. One group of CVs called "Z Cam-type DNe" are believed to be the intermediate systems between DNe and NLs, since they show occasionally "standstills" having constant luminosity intermediate between outburst maxima and minima. It is believed that the variations of mass transfer rates are necessary to reproduce this kind of phenomena (e.g., Meyer, Meyer-Hofmeister 1983). There is at least one attempt to reproduce them by the fluctuations of viscosity instead of those of transfer rates, but they failed to generate long-lasting standstills and several consecutive outbursts (Ross, Latter 2017).

Simonsen (2011) noticed the presence of two unusual DNe which show repeated outbursts to standstills terminated by brightening (instead of fading in ordinary Z Cam stars). After that, Szkody et al. (2013) called these objects "anomalous Z Cam stars" and discussed the potential relation with small outbursts in NLs. Recently, Kato (2019) found three more objects showing the same behavior as that reported by these authors and named these objects "IW And-type stars", and has pointed out that more or less regular repetition of standstills terminated by brightening are common to these objects (see also Sec. 2). He further suggested the presence of a previously unknown type of limit-cycle oscillation in IW And-type stars.

Hameury, Lasota (2014) explored for the first time the cause of "anomalous Z Cam phenomenon" and proposed a model in which the variation in mass transfer rates from the secondary star is responsible for that phenomenon. However, there is no positive evidence for triggering modulations of transfer rates, and also, the brightening of bright spots, which should occur simultaneously with the sudden increase of transfer rates, are observationally undetected (Honeycutt 2001; Schlegel, Honeycutt 2019). Hameury, Lasota (2014) have sought the cause of the light variations of IW And-type stars in the outside of the disk, i.e., enhanced mass transfer from the secondary star. However, it would be more preferable if a previously unknown type of limit-cycle oscillation is found within the disk, as suggested by Kato (2019). Since the standard thermal-viscous instability model is very unlikely to produce standstills terminated by brightening, we need to seek some new aspects not considered in the standard disk instability model. To this end, recently, time-resolved optical photometry gave us a clue, the detections of negative superhumps in some of IW And stars.

Gies et al. (2013) and Armstrong et al. (2013) observed negative superhumps in some DNe that were identified to be IW And-type stars later (e.g., Kato 2019). The negative superhumps are photometric light modulations with periods slightly shorter than the orbital period, i.e., they show the negative excess to the orbital period (Harvey et al. 1995), and they are interpreted as the transit of the bright spot on the tilted and/or warped disk misaligned to the orbital plane, which experiences retrograde nodal precession (Wood et al. 2000; Murray et al. 2002; Wood, Burke 2007). Importantly, this interpretation means that the gas stream often flows into the inner part in tilted disks, while it always collides with the outer edge of the disk in non-tilted disks. In fact, Kato (2019) has suggested that standstills in IW Andtype DNe may somehow be maintained as the inner part of the disk stays in the hot state, while the outer part is in the cool state, and that the thermal instability starting from the outer part of the disk terminates standstills. The mass input in the tilted disk might achieve such a new limit cycle by keeping the inner disk hot during standstills in IW And-type DNe.

Motivated by this suggestion, we study the disk instability model in the case of tilted accretion disks. The aim of the present study is twofold: one is to investigate how the thermal instability works in tilted accretion disks and another is to see to what extent the thermal instability in the tilted disk could explain the essential features of light variations in IW And-type DNe. In Sec. 2, we introduce the observational light variations in IW And-type stars and specify their essential features, which we aim at reproducing. In Sec. 3, we give our assumptions and the method of our simulations in time evolution of the one-dimensional disk. In Sec. 4, we calculate the patterns of mass input in tilted disks, a key of our simulations, and list the calculated models. In Sec. 5, we present the numerical simulations. We discuss our results in Sec. 6, comparing them with observations. Finally, we give our summary in Sec. 7 .

\section{Observational light variations in IW And-type stars}

In these couple of years, we have obtained much more knowledge about the light variations in IW And stars than we did when the first example of the IW And-type DNe was recognized. Here we introduce briefly what the essential features of the IW And-type light variations are on the basis of the latest observations.

IW And-type stars commonly show repetitive light variations as pointed out in Kato (2019). Figure 1 displays the typical IW And-type light variations of IM Eri ${ }^{1}$, which was identi-

\footnotetext{
${ }^{1}$ Kato et al. (2019) report in detail this kind of light variations of this object, which was observed in 2018.
} 


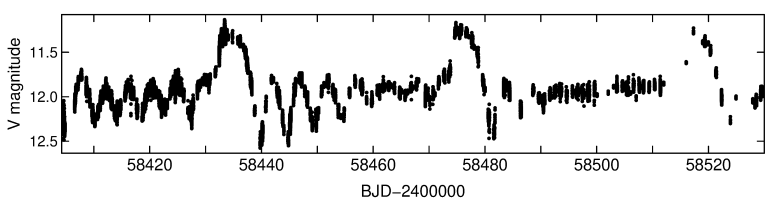

Fig. 1. An example of the IW And-type phenomenon from a part of the 2018 light curves of IM Eri, which are obtained by a campaign led by Variable Star Network (VSNET). All of the IW And-type phenomenon of this object in 2018 is presented in Kato et al. (2019). Here, BJD is barycentric Julian date.

fied as an IW And-type star by Kato (2019). The most characteristic feature of the typical IW And-type light variations is "quasi-standstills" terminated by brightening, and they are one of the essential features of light variations in IW And stars. Here quasi-standstills are the state in intermediate brightness with (damping) oscillatory variations, which are different from standstills with almost constant luminosity in normal Z Cam stars. We call this the IW And-type phenomenon hereafter. Deep luminosity dips occasionally follow brightening (see also Figure 2). The amplitudes of brightening are typically less than $1 \mathrm{mag}$. The averaged interval between brightening is $\sim 50$ days, but its length never remains in one constant value within one object.

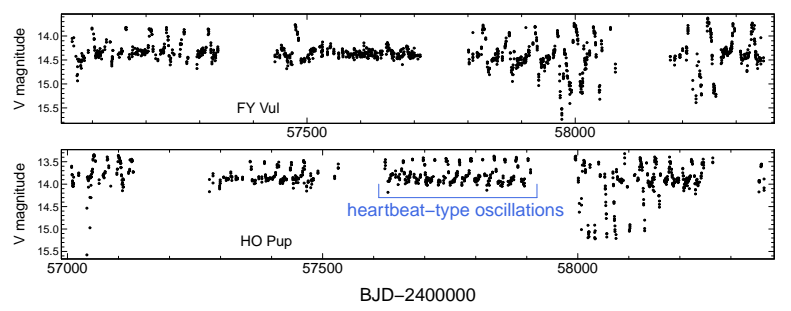

Fig. 2. A wide variety in long-term light curves of FY Vul and HO Pup, IW And-type stars. We have obtained the data from ASAS-SN data archive (Davis et al. 2015).

Moreover, long-term photometric surveys for optical transients revealed that diverse light variations can be observed on long timescales even within one IW And star. Figure 2 illustrates long-term light curves of two IW And stars, FY Vul and HO Pup, which were recognized as IW And-type DNe by one of the authors (T.K. 2018, vsnet-chat $8101^{2}, 8162^{3}$ ). It is another essential feature of the light variations in IW And stars that they would alternate within one object between the IW And-type phenomenon, Z Cam-type standstills, normal dwarf-nova outbursts, and heartbeat-type oscillations on timescales of $\sim 100$ 1000 days. The averaged optical luminosities among those different types of light variations are almost constant, and thus the time variations in mass transfer rates are unlikely for the cause

\footnotetext{
${ }^{2}<$ http://ooruri.kusastro.kyoto-u.ac.jp/mailarchive/vsnet-chat/8101>

${ }^{3}<$ http://ooruri.kusastro.kyoto-u.ac.jp/mailarchive/vsnet-chat/8162>
}

of their diversity. Here heartbeat-type oscillations indicated in the lower panel of Figure 2 seem to be a new-type light variability, but may be common in IW And stars, because similar behavior has been detected also in other objects (e.g., Ramsay et al. 2016). We use the term "heartbeat-type oscillations" here, since this type of variability resembles "heartbeat oscillations" observed in GRS 1915+105, a famous black-hole binary, which are considered to be caused by limit-cycle accretion triggered by the radiation-pressure instability (Fender, Belloni 2004; Watarai, Mineshige 2003; Neilsen et al. 2011). (This term should not be confused with the pulsation phenomenon of "heartbeat stars" discovered by Kepler data (Thompson et al. 2012).)

\section{Method of numerical calculations for time-dependent disks}

\subsection{Basic assumptions of our model}

In this study, we consider how the problem of thermal-viscous disk instability in $\mathrm{CVs}$ could be applied to the tilted disks. Generally speaking, if the disk is tilted, it would have warped structures and it would experience retrograde nodal precessions. In the binary frame of reference, the disk structure would vary with the period of negative superhumps, i.e., the synodic period between the orbital period and the precession period. To study such complicated problems, we have to perform a full 3-dimensional hydrodynamical simulations of a nonaxisymmetric disk whose structures change with time, which is not an easy task.

However, we think that one of the most important effects of the disk tilt on the problem of thermal-viscous disk instability is that the gas stream from the secondary star will penetrate deeply into the inner part of disk, in contrast to the standard case of a non-tilted disk where the gas stream arrives mostly in the outer disk edge. In this paper, we concentrate ourselves to study this particular effect of the tilted disk. For this purpose, we adopt various simplifying assumptions as far as possible. We first assume that the tilted disk is not warped and rigidly tilted and we take this tilted plane misaligned to the orbital plane as our frame of reference. We further assume that the disk is axi-symmetric in this frame of reference and we choose the cylindrical coordinates, $r-z$ in this plane, where $r$ is the distance from the central white dwarf and $z$ is the distance perpendicular to this plane. By these simplified assumptions our problem reduces to the same problem as the standard case of a non-tilted disk, and the effects of tilt enter only through the mass supply pattern different to that in the standard case. In fact, the standard case of a nontilted disk is regarded as one of our special cases with the zero tilt angle.

Furthermore, we do not discuss any problems of time variations shorter than the negative-superhump period and we aver- 
age the mass supply pattern over that period (see also Sec. 4.1). This is because we focus on the light variations on timescales of days much longer than the period of negative superhumps.

\subsection{Basic equations for a viscous disk}

We calculate the time evolution of a geometrically-thin and axisymmetric accretion disk with an assumption of a co-planar but tilted disk, basically in the same way as in Ichikawa, Osaki (1992). It is noted here that since the original computer code by Ichikawa, Osaki (1992) does not exist any more, we have newly written our simulation code from the beginning in this study, following that paper.

We assume that the disk in our consideration is geometrically thin and that it is in hydrostatic equilibrium in the vertical $z$-direction. We adopt the one-zone model in the $z$-direction. Here, we use the cylindrical coordinates, $(r, \phi, z)$, with $z$-axis being the disk rotation axis. The surface density, $\Sigma$, is then given in the one-zone model by

$\Sigma=\int_{-\infty}^{\infty} \rho d z=2 \rho_{\mathrm{c}} H$,

where $\rho$ is the density, $H$ is the scale height, i.e., the half thickness of the disk which is given later by equation (6), and $\rho_{\mathrm{c}}$ is the density at the disk mid-plane, respectively. Hereafter we express the variables at the disk mid-plane with the subscript "c". We use vertically-integrated basic equations for a viscous disk: the equations for conservation of mass, angular momentum, and energy, by adopting vertically the one-zone model.

The equation for mass conservation is

$\frac{\partial(2 \pi r \Sigma)}{\partial t}=\frac{\partial \dot{M}}{\partial r}+s$,

where $\Sigma(r, t)$ is the surface density at a radial position, $r$, from the central white dwarf and time, $t$, in the unit of $\mathrm{g} \mathrm{cm}^{-2}$, and $\dot{M}(r, t)$ is the mass accretion rate in the unit of $\mathrm{g} \mathrm{s}^{-1}$, which is defined by $-2 \pi r \Sigma v_{r}$, and $v_{r}$ is the radial velocity of the gas flow in the disk, and the source term, $s(r)$, is the mass supply rate per unit distance via the stream from the secondary star at a given radius of the accretion disk, respectively. We assume the source term to be time independent in the present study, and give our formulation for $s(r)$ in the case of tilted disks in Sec. 4.1.

The equation for angular momentum conservation is

$\frac{\partial(2 \pi r \Sigma h)}{\partial t}=\frac{\partial(\dot{M} h)}{\partial r}-\frac{\partial}{\partial r}\left(2 \pi r^{2} W\right)-D+h_{\text {LS }} s$,

where $W$ is the vertically integrated viscous stress, $D$ is the tidal torque exerted by the secondary star, and $h=\sqrt{G M_{1} r}$ and $h_{\mathrm{LS}}=\sqrt{G M_{1} r_{\mathrm{LS}}}$ are the specific angular momentum of the disk matter and that of the gas stream from the secondary star at a given radius, respectively. Here $M_{1}$ is the mass of the primary star, and $G$ is the gravitational constant, respectively. We assume that the specific angular momentum of the gas stream is conserved to be that at the Lubow-Shu radius, $r_{\mathrm{LS}}$ (Lubow, Shu 1975). Here we consider only $r \phi$-component of viscous stress tensor, expressed by $w_{r \phi}$, and hence, $W \equiv \int-w_{r \phi} d z$. We take $\phi$ as the azimuthal angle in the cylindrical coordinates. Here $w_{r \phi}$ includes the shear viscosity coefficients, and is expressed as $-3 \rho \nu \Omega / 2$, by using the kinematic viscosity $\nu$. By adopting $\alpha$-prescription proposed by Shakura, Sunyaev (1973), $w_{r \phi}$ is represented as $-\alpha P$, where $P$ is the pressure and $\alpha$ is the viscosity parameter formulated in Sec. 3.4, respectively. The vertically integrated viscous stress, $W$, is then expressed under the one-zone approximation as follows:

$W=\frac{3}{2} \nu \Sigma \Omega=2 H \alpha P_{\mathrm{c}}=\alpha \frac{\mathscr{R}}{\mu_{\mathrm{c}}} \Sigma T_{\mathrm{c}}$,

where $T$ is the temperature, $\Omega=\sqrt{G M_{1} / r^{3}}$ is the Keplerian angular velocity, $\mathscr{R}$ is the gas constant, and $\mu$ is the mean molecular weight, respectively. We do not consider the radiation pressure, and $P$ represents the gas pressure defined as

$P=\frac{\mathscr{R}}{\mu} \rho T$.

Here the scale height of the disk, $H$, is defined as

$H=\frac{1}{\Omega} \sqrt{\frac{\mathscr{R}}{\mu_{\mathrm{c}}} T_{\mathrm{c}}}$

by using $\frac{P_{\mathrm{c}}}{\rho_{\mathrm{c}}}=(H \Omega)^{2}$ on the basis of the one-zone approximation.

As for the tidal torque, we adopt the following expression by Smak (1984):

$D=c \omega r \Sigma \nu\left(\frac{r}{a}\right)^{5}$,

where $a$ is the binary separation. By substituting equation (2) to equation (3), we obtain the following relation:

$\dot{M} \frac{\partial h}{\partial r}=\frac{\partial}{\partial r}\left(2 \pi r^{2} W\right)+D+\left(h-h_{\mathrm{LS}}\right) s$.

The equation for energy conservation is

$$
\begin{array}{r}
C_{\mathrm{P}}\left[\frac{\partial}{\partial t}\left(2 \pi r \Sigma T_{\mathrm{c}}\right)-\frac{\partial}{\partial r}\left(\dot{M} T_{\mathrm{c}}\right)-2 \pi \Sigma \nu_{\mathrm{th}} \frac{\partial\left(r F_{r}\right)}{\partial r}-s T_{\mathrm{c}}\right] \\
=2 \pi r\left(Q^{+}-Q^{-}\right),
\end{array}
$$

where $T_{\mathrm{c}}(r, t)$ is the temperature at the mid-plane of the disk in the unit of $\mathrm{K}$. Also, $Q^{+}$and $Q^{-}$represent the heat generation and the radiative loss from the disk surface per unit surface area, respectively. The thermodynamic quantities of $\mu$ in equation (4) and the specific heat at constant pressure, $C_{\mathrm{P}}$, are evaluated at the mid-plane disk. We adopt the chemical composition of population I stars: $X=0.70, Y=0.27$, and $Z=0.03$, where $X$, $Y$, and $Z$ are the hydrogen content, the helium content, and the metallicity, respectively. In calculating the thermodynamic quantities, we take into account the ionization of hydrogen, and the first and second ionizations of helium, and the dissociation of the $\mathrm{H}_{2}$ molecule in the same way as described in Paczyński (1969). The second, third, and fourth terms in the left-hand 
side of equation (9) represent those of the advection, the thermal diffusion, and the input energy by the gas stream from the secondary star, respectively. In the term of the thermal diffusion $F_{r}$ is the temperature gradient expressed as $\partial T_{\mathrm{c}} / \partial r$, and $\nu_{\text {th }}$ signifies the thermal diffusivity. Here we consider the dynamical diffusivity only, and $\nu_{\text {th }}$ is given by $2 W / \Omega \Sigma$ (Meyer 1984; Mineshige 1986). In the fourth term, we assume the gas stream has the same temperature as the pre-existing disk matter for simplicity. We note here that all variables appearing in these equations are now calculated once the two quantities, $\Sigma$ and $T_{\mathrm{c}}$, at a given $r$ are known.

\subsection{Heating and cooling functions}

To solve the energy conservation denoted by equation (9), we need to express $Q^{+}$and $Q^{-}$as the functions of $T_{\mathrm{c}}$ and $\Sigma$. In this study, we consider the following three terms as the heating source $Q^{+}$:

$Q_{1}^{+}=\frac{3}{2} W \Omega$

$Q_{2}^{+}=D \frac{\Omega-\omega}{2 \pi r}$,

$Q_{3}^{+}=\frac{\beta}{2} \frac{G M_{1}}{r} \frac{s}{2 \pi r}$.

Here, $Q_{1}^{+}, Q_{2}^{+}$, and $Q_{3}^{+}$represent the shear viscous heating, the tidal dissipation, and the energy dissipation of the gas stream from the secondary star, respectively. The fraction of energy dissipation $\beta$ depends on the radius, and is calculated in Sec. 4.1. The cooling rate is expressed as $Q^{-}=2 F$. The radiative loss function $F$ is obtained by integrating the vertical structure of the convective accretion disk as calculated in e.g. Mineshige, Osaki (1983); Hameury et al. (1998). We, however, use simplified interpolation formulae below in this study. The thermal equilibrium curve of the disk is composed of three branches: a hot branch where hydrogen is fully ionized, an intermediate branch where hydrogen is partially ionized, and an optically-thin cool branch where hydrogen is neutral.

In the one-zone model, the radiative flux from the opticallythick disk is given as

$F=\frac{16 \sigma T_{\mathrm{c}}^{4}}{3 \kappa_{\mathrm{c}} \rho_{\mathrm{c}} H}$,

where $\sigma$ is the Stefan-Boltzmann constant, and $\tau=\kappa_{\mathrm{c}} \rho_{\mathrm{c}} H$ is the optical depth of the disk, respectively. As for the hot branch, the opacity $(\kappa)$ is given by Kramers law of ionized gas (equation (3.14) in Cannizzo et al. 1988) as follows:

$\kappa=2.8 \times 10^{24} \rho T^{-3.5} \mathrm{~cm}^{2} \mathrm{~g}^{-1}$.

By combining equations (6), (13), and (14), the radiative flux at the hot branch is obtained as

$\log F_{\text {hot }}=8 \log T_{\mathrm{c}}-\log \Omega-2 \log \Sigma-0.5 \log \mu_{\mathrm{c}}-23.405 .(15)$

By contrast, we adopt the opacity of the negative hydrogen

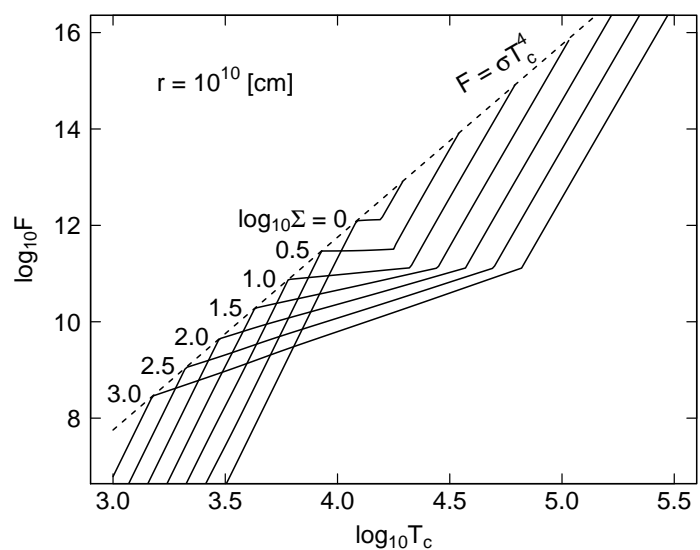

Fig. 3. The relation between the temperature and the radiative flux as for 6 different values of $\Sigma$ at $r=10^{10} \mathrm{~cm}$, calculated by equations (15), (18), and (20). The dashed line represents $F=\sigma T_{c}^{4}$.

given by the following interpolation formula in the opticallythin region on the basis of the Cox and Stewart opacity (Cox, Stewart 1969) as shown in Fig. 2 in Cannizzo, Wheeler (1984):

$\kappa=5.13 \times 10^{-19} \rho^{0.62} T^{5.8} \mathrm{~cm}^{2} \mathrm{~g}^{-1}$,

where we do not consider the molecular opacity. The radiative flux in the optically thin case is then as follows:

$F=\tau \sigma T_{\mathrm{c}}^{4}$

From equations (6), (16), and (17), the radiative flux at the cool branch is obtained as

$\log F_{\text {cool }}=9.49 \log T_{\mathrm{c}}+0.62 \log \Omega+1.62 \log \Sigma+0.31 \log \mu_{\mathrm{c}}-25.48$

As for the intermediate branch, we assume that the cool branch extends to the critical temperature $T_{\mathrm{A}}$ at which $F=\sigma T_{\mathrm{A}}^{4}=F_{\mathrm{A}}$, and that the hot branch extends to $T_{B}$ at which the radiative flux is approximately represented by

$\log F_{\mathrm{B}}=11+0.4 \log \left(\frac{2.0 \times 10^{10}}{r}\right)$.

Here, $F_{B}$ is equal to $F_{A}$ if $F_{B}<F_{A}$. The radiative flux at the intermediate branch is as follows:

$\log F_{\text {int }}=\left(\log F_{A}-\log F_{B}\right) \log \frac{T_{\mathrm{c}}}{T_{B}} / \log \frac{T_{A}}{T_{B}}+\log F_{B}$.

Some examples of the relation between the temperature and the radiative flux are given in Figure 3.

\subsection{Radial dependence of the viscosity parameter}

The past studies suggest the necessity of $r$ dependence of $\alpha$ in equation (4) in quiescence and/or the difference of $\alpha$ between 
the hot and cool branches in order to reproduce the observational amplitudes of outbursts and frequent outside-in outbursts (Mineshige, Wood 1989; Hameury et al. 1998; Cannizzo 1993). We therefore adopt the following formulation:

$$
\begin{aligned}
\log \alpha & =\frac{1}{2}\left(\log \alpha_{\text {hot }}-\log \alpha_{\text {cool }}\right)\left[1-\tanh \left(\frac{4-\log T_{\mathrm{c}}}{0.4}\right)\right] \\
& +\log \alpha_{\text {cool }} \\
\alpha_{\text {cool }} & =0.03\left(\frac{r}{r_{\text {tidal }}}\right)^{0.5}, \\
\alpha_{\text {hot }} & =0.3
\end{aligned}
$$

where $\alpha$ is the function of $r$ and $T_{\mathrm{c}}$. The $\alpha$ value becomes close to $\alpha_{\text {cool }}$ in the cool state, and becomes close to $\alpha_{\text {hot }}$ in the hot state. We now have the definitions of variables necessary for our simulations except for those of $s(r)$ and $\beta$. We also obtain the the relation between the surface density and the effective temperature, which is called the thermal equilibrium curve, as in Figure 4. In this figure, we do not consider $Q_{3}^{+}$because $s(r)$ depends on the mass input pattern described in Sec. 4.1.

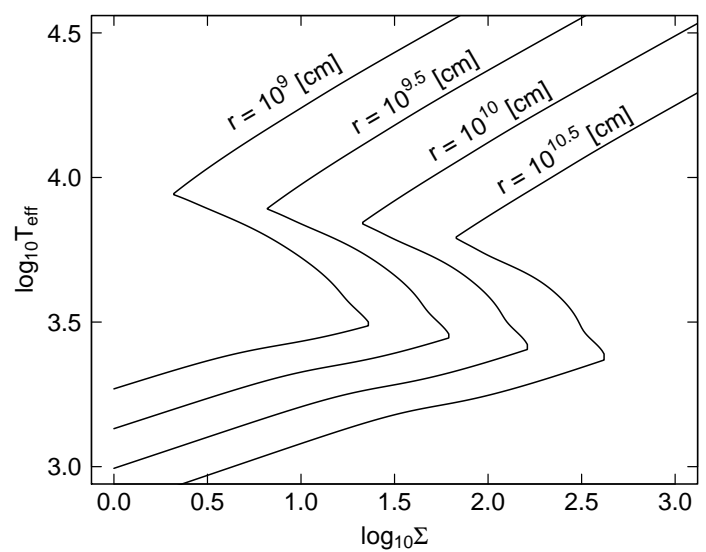

Fig. 4. The thermal equilibrium curves at $r=$ $10^{9}, 10^{9.5}, 10^{10}$, and $10^{10.5} \mathrm{~cm}$, calculated by $2 F=Q_{1}^{+}+Q_{2}^{+}$. The vertical axis represents the effective temperature. Here we use the binary parameters of $U$ Gem, given in Sec. 4.2.

\subsection{Finite-difference scheme}

The finite-difference scheme used in this paper is the same as that described in Ichikawa, Osaki (1992). We treat the conservation of the total angular momentum of a disk in the scheme, by letting the outer disk edge variable. Its detailed description is given in Appendix 1. We divide the accretion disk into $N$ concentric annuli. We define the interface between $i$-th annulus and $(i+1)$-th annulus by $r_{i}$. The number of interfaces is then $N+1$ $(i=0,1,2, \ldots, N)$. The inner boundary of the disk is now given by $r_{0}$ and the outer boundary is given by $r_{N}$. A special treatment is needed for the outermost annulus and its treatment is the same as in Ichikawa, Osaki (1992). In short, the outer edge of the disk, $r_{N}$, is varied in such a way to conserve the total mass and the total angular momentum of the disk. The largest radius of the disk is the tidal truncation radius, $r_{\text {tidal }}$. When the disk tries to expand beyond the tidal truncation radius, we fix the disk radius at the tidal truncation radius by removing the extra angular momentum from the disk. In our calculations, 200 meshes are used at most. The details of the scheme and the radial distribution of meshes are described in Appendix 1.

\section{Mass input patterns and calculated models}

\subsection{Mass input from the secondary star to a tilted disk}

As described in Sec. 3.1, the key of our simulations is to consider how outburst behavior changes when we vary the mass supply pattern, $s(r)$, in tilted disks where the gas stream enters not only the disk outer edge but also the inner disk. The location of a bright spot on the disk surface varies depending on the position of the secondary star against the tilted disk. To treat this problem, we have estimated which radius of the tilted disk the gas-stream trajectory first collides with, while the tilted disk rotates half around against the secondary during the half period of negative superhumps. We then obtain the time-averaged mass input pattern in the $r$-direction, $s(r)$. Finally we have formulated three representative mass input patterns in the low tilt case, the moderate tilt case, and the high tilt case, respectively.

We have computed the ballistic trajectory of a particle at first by solving restricted three-body problem with the binary parameters of U Gem. We have used the equations (1) and (2) in Flannery (1975), which represent the equation of motion in a co-rotating frame with the binary. Here we take $x-y$ plane as the orbital plane of the binary, and $z^{\prime}$-direction perpendicular to the orbital plane. We set the primary and the secondary on $x$-axis as point masses. The gas stream comes into the primary Roche lobe via the Lagrangian point (the L1 point), and the movement of a particle is governed by the gravitational fields of the primary and the secondary, and the Coriolis force. We assume the initial velocity of a particle toward $x$-direction is 0.03 , which is normalized by the orbital velocity of the binary. This value is consistent with the sound speed of the atmosphere of the secondary star (Lubow, Shu 1975). The resultant gas-stream trajectory is shown as the thick black line in Figure 5.

We have next determined the geometry of the disk. Here we assume that the tilted disk has the thickness expected from the steady standard disk for convenience (Shakura, Sunyaev 1973) as follows:

$$
\frac{H}{r}=1.72 \times 10^{-2} \alpha^{-1 / 10} \dot{M}_{16}^{3 / 20}\left(\frac{M_{1}}{M_{\odot}}\right)^{3 / 8} r_{10}^{1 / 8}\left[1-\left(\frac{r_{\text {in }}}{r}\right)^{1 / 2}\right]^{3 / 5}(24)
$$




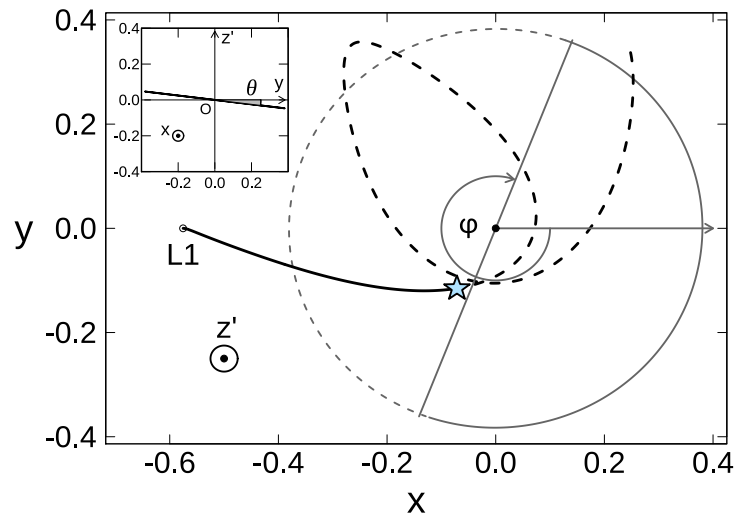

Fig. 5. Trajectory of the gas stream from the secondary star, which moves on the $x-y$ plane. The grids are normalized by the binary separation. The L1 point is located at $(x, y)=(0,-0.575)$. The white dwarf and the secondary are located at $(x, y)=(0,0)$ and $(-1,0)$, respectively. The black point represents the center of the white dwarf. Here we adopt the tilt angle, $\theta=7$ deg, and we show a particular case of $\varphi=289.6 \mathrm{deg}$, where $\varphi$ is the angle by which the nodal line (shown by the diametric line) makes with the $x$-axis and it is counted clockwise. The solid thick line represents the trajectory, and the mark 'star' is the first crossing point of the gas-stream trajectory against the surface of the tilted disk. The thick dashed line represents the trajectory of gas stream after that, if no collision had occurred. The grey thin line represents the contour of the tilted mid-plane disk. The solid grey line means that the mid-plane is above the $x-y$ plane, and the dashed grey line means that it is below the $x-y$ plane. In the small inlet, we indicate the $y-z^{\prime}$ plane and the tilt angle $\theta=7$ deg when $\varphi=0$.

where $r_{10}$ is $r$ in the unit of $10^{10} \mathrm{~cm}$, and $\dot{M}_{16}$ is $\dot{M}_{\text {tr }}$ in the unit of $10^{16} \mathrm{~g} \mathrm{~s}^{-1}$, respectively. Here $\dot{M}_{\text {tr }}$ is the mass transfer rate from the secondary star. We set $\dot{M}_{16}$ and $\alpha$ in equation (24) to be 10 and 0.3 , respectively, and assume that the outermost radius of the disk is the tidal truncation radius. In reality, the thickness and the size of the disk vary with time but we do not consider such effects here for simplicity.

By using the trajectory and the disk geometry that we have prepared, we have calculated the first-crossing points between the gas stream and the tilted disk, once we fix a tilt angle $(\theta=$ 7 deg in Figure 5). The tilted accretion disk rotates clockwise around the white dwarf (i.e., the $z^{\prime}$-axis) in the co-rotating frame with the binary, and it takes one period of negative superhumps for one rotation. The rotational angle is denoted as $\varphi$ and it is defined as the angle from the positive $x$-axis to the nodal line of the tilted disk and measured clockwise. It varies according to $\varphi=\omega_{\mathrm{nSH}} t+\varphi_{0}$, where $\omega_{\mathrm{nSH}}$ is the angular velocity of the tilted disk, and $\varphi_{0}$ is the initial value which we choose when the gas stream collides with the nodal line of the tilted disk at the disk edge. We show the definitions of $\varphi$ and $\theta$ in Figure 5 .

The gas stream moves on the $x-y$ plane. For example, the gas stream collides with the tilted disk surface at the mark 'star' in Figure 5 when $\varphi=289.6$ deg and $\theta=7$ deg. When the tilt

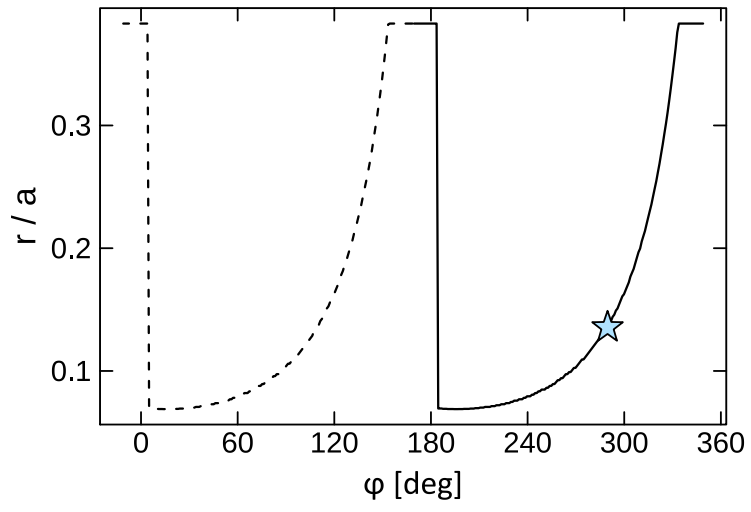

Fig. 6. Radial coordinates of the first crossing points of the gas stream from the secondary star when a tilted disk rotates around the $z^{\prime}$-axis during one period of negative superhumps in the case of $\theta=7 \mathrm{deg}$. The horizontal axis represents the rotational angle $\varphi$ of the accretion disk in the co-rotating frame with the binary. The vertical axis represents the radial distance of the crossing points from the white dwarf, which is normalized by the binary separation. The mark 'star' corresponds to that in Figure 5. The dashed line means the gas stream enters the back face of the tilted disk, and the solid line means it enters the front face, respectively.

angle is small, the crossing point is slightly deviated from the nodal line since we assume the disk has a finite thickness. We have calculated and recorded the radial distance of the crossing points from the white dwarf during one rotation of the tilted disk, by incrementing $\varphi$ by 1 deg from $\varphi_{0}$ to $\varphi_{0}+2 \pi$. The results for $\theta=7 \mathrm{deg}$ are shown in Figure 6. Here we show the results as the dashed line if the gas stream collides on the back face of the disk, and as the solid line if the gas stream collides on the front face where we assume that the observer looks at the disk from above, for instance, with an inclination angle $i=$ 45 deg. Since these two cases (i.e., the back face and the front face) are the same with respect to the radial distribution, we have calculated the crossing points only when the gas stream collides with the disk on the front face during a half period of negative superhumps.

We have repeated this kind of calculations over the range of $\theta$ from 1 to $30 \mathrm{deg}$ by $1 \mathrm{deg}$. We have then estimated how frequent each region of the accretion disk in the $r$-direction receives the gas stream as shown in Figure 7. Here we prepare three examples with three different tilt angles. These three panels bring the time-averaged mass input patterns themselves. It has turned out the patterns hardly depend on the tilt angle above $15 \mathrm{deg}$. The corresponding tilt angles to these three panels are 3, 7, and 15 deg in Figure 7 when we assume the standard-disk geometry in the hot disk for convenience. However, the actual disk does not always stay in the hot state and the disk is mostly thinner than in the case we calculated. Therefore these tilt angles do not represent real ones and are very much over-estimated. In 
what follows, we refer these three mass input patterns as those in the slightly-tilted disk, in the moderately-tilted disk, and in the highly-tilted disk, respectively, and we do not use these tilt angles any more. The mechanism of the disk tilt is still unknown (see also Sec. 6.2) and the tilt angle of the accretion disk is hard to measure. There is no negative observational evidence about high tilt angles. That is the reason why we consider the high tilt case as well. We see the gas stream often reaches the vicinity of $r_{\text {input,min }}$ in the high tilt case, while it is mostly intercepted at the outer edge in the low tilt case. Here $r_{\text {input,min }}$ signifies the innermost radius where the gas stream from the secondary star reaches.

To implement three mass input patterns shown in Figure 7 to our simulations, we have formulated them by dividing the accretion disk into the three regions as in each panel of Figure 8. We set the three values of $\dot{m}_{\mathrm{in}}=\dot{M}_{\mathrm{in}} / \dot{M}_{\mathrm{tr}}$, $\dot{m}_{\text {mid }}=\dot{M}_{\text {mid }} / \dot{M}_{\text {tr }}$, and $\dot{m}_{\text {edge }}=\dot{M}_{\text {edge }} / \dot{M}_{\text {tr }}$, respectively. Here, $\dot{M}_{\mathrm{tr}}$ is the mass transfer rate from the secondary star, and $\dot{M}_{\text {in }}, \dot{M}_{\text {mid }}$, and $\dot{M}_{\text {edge }}$ are the mass input rates into the region 1,2 , and 3 , respectively. We have calculated these values according to the results shown in Figure 7, and show them in Figure 8. Here we also display the mass input pattern of the non-tilted standard case in its upper-left panel. In this case, both of $\dot{m}_{\text {in }}$ and $\dot{m}_{\text {mid }}$ are zero. We thus see the difference in our simulations between the non-tilted case and the tilted cases is only the mass supply pattern. We next have approximately expressed the source term $s(r)$ as follows:

$$
\begin{array}{r}
s(r)=\frac{2 \dot{M}_{\text {in }}}{\left(r_{\mathrm{LS}}-r_{\text {input } \text { min }}\right)^{2}}\left(r-r_{\text {input }, \text { min }}\right) \\
\left(r_{\text {input }, \text { min }} \leq r \leq r_{\mathrm{LS}}\right), \\
s(r)=\frac{\dot{M}_{\text {mid }}}{\log \frac{r_{N-N_{\mathrm{S}}}}{r_{\mathrm{LS}}} \frac{1}{r} \quad\left(r_{\mathrm{LS}} \leq r \leq r_{N-N_{\mathrm{S}}}\right),} \\
s(r)=\frac{\dot{M}_{\mathrm{edge}}}{N_{\mathrm{S}} \mathrm{dr}} \quad\left(r_{N-N_{\mathrm{S}}} \leq r \leq r_{N}\right),
\end{array}
$$

for $\mathrm{dr}_{\mathrm{N}} \geq \mathrm{dr}$. Here $N_{\mathrm{S}}$ is 10 , and $\mathrm{dr}_{\mathrm{N}}$ is the width of the outermost radius, respectively. If $r<r_{\text {input,min }}, s(r)$ is 0 . When $\mathrm{dr}_{\mathrm{N}}$ is less than dr that we define in equation (A14) in Appendix 1, the boundary between the regions 2 and 3 becomes $r_{N-N_{S}-1}$ instead of $r_{N-N_{S}}$ in equations (26) and (27). We have chosen the triangular distribution as for the mass supply rate at the region 1 in Figure 8 to avoid numerical difficulties (see discussion in Sec. 6.3).

We have also estimated the value of $\beta$ in equation (12), which depends on the radius where the gas stream collides with the tilted disk, by calculating the relative speed between the gas stream and the disk matter rotating around the central white dwarf with the Keplerian velocity. If we write the relative velocity as $v_{\text {rel }}$, the $\beta$ value is defined as follows:

$\beta=0.5 \frac{v_{\mathrm{rel}}^{2} / 2}{G M_{1} / 2 r}$.
Here, we assume that the half of the thermally dissipated energy by the gas stream is radiated locally as a bright spot, and the other half is used to heat the disk. The resultant $\beta$ value is given in Figure 9.

\subsection{Model parameters and lists of calculated models}

We first specify the binary parameters for our simulations. Since the aims of the present study is to examine how outburst properties depend on the mass input pattern and the tilted disk could reproduce the essential features of the light variations in IW And-type stars, it is preferable to adopt the typical binary parameters in IW And-type DNe in our simulations. Unfortunately, the binary parameters of IW And stars are not well known, but we have some knowledge that their orbital periods fall on a wide range of $0.14-0.48 \mathrm{~d}$ above the period gap (Rodríguez-Gil et al. 2007; Armstrong et al. 2013; Szkody et al. 2013; Echevarría, Michel 2007; Ringwald 1994; Cieslinski et al. 1998; Gies et al. 2013; Thorstensen et al. 2004). We thus can presume that IW And stars would have similar binary parameters to those of $\mathrm{U}$ Gem stars having a similar range of orbital periods except for the mass transfer rate. Under such a circumstance, we basically adopt the binary parameters appropriate for U Gem in the following calculations, because the period of $U$ Gem is within the period range of IW And stars mentioned above and because the binary parameters of U Gem are the best known among DNe. Besides, we have made additional simulations with the binary parameters of KIC 9406652 in order to confirm that our main results do not depend very much on the binary parameters used. KIC 9406652 is only one object whose mass ratio was measured among IW And-type stars (Gies et al. 2013).

The binary parameters of $\mathrm{U}$ Gem adopted in this study is as follows: the orbital period $\left(P_{\text {orb }}\right)$ is $0.176906 \mathrm{~d}$, the whitedwarf mass $\left(M_{1}\right)$ is $1.18 M_{\odot}$, the mass of the secondary $\left(M_{2}\right)$ is $0.55 M_{\odot}$, the binary separation $(a)$ is $1.115 \times 10^{11} \mathrm{~cm}$, the tidal truncation radius $\left(r_{\text {tidal }}\right)$ is $0.383 a$, the Lubow-Shu radius $\left(r_{\mathrm{LS}}\right)$ is $0.117 a$, and the inner edge of the disk $\left(r_{0}\right)$ is $5 \times 10^{8} \mathrm{~cm}$ (i.e., at the surface of the primary white dwarf), respectively (Anderson 1988). Additionally, we set $c \omega$ in equation (7) to be 0.4 as a realistic value according to Ichikawa, Osaki (1994). According to Lubow, Shu (1975), $r_{\text {input,min }}$ is estimated to be $0.069 a$ when the binary mass ratio $(q)$, which is defined as the mass ratio of the secondary with respect to the primary, is 0.47 .

We next need to specify the mass transfer rate, $\dot{M}_{\mathrm{tr}}$. Since one of our aims in this paper is to try to understand the outbursts of IW And-type DNe, the mass transfer rates, $\dot{M}_{\mathrm{tr}}$, discussed mainly here are rather high: $10^{16.75} \mathrm{~g} \mathrm{~s}^{-1}$ and $10^{17} \mathrm{~g} \mathrm{~s}^{-1}$ near the critical mass transfer rate $\dot{M}_{\text {crit }}$, above which the disk becomes in the hot steady state. However, we also show the results of the cases with other two values of mass transfer rates in 

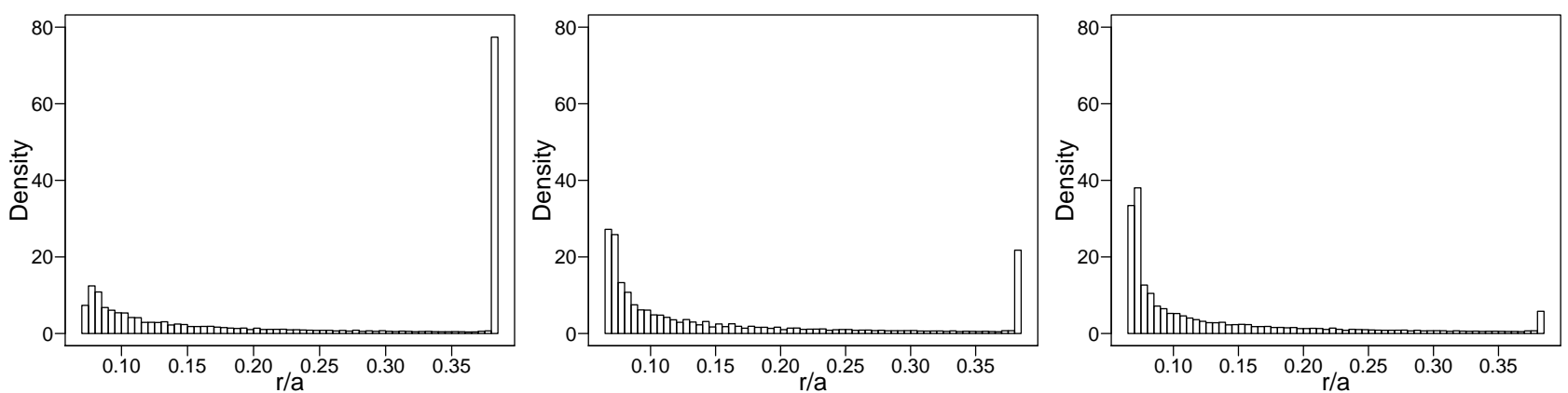

Fig. 7. These panels show how frequent the gas stream enters each annulus during the period of negative superhumps, i.e., while the tilted disk rotates once against the secondary star. (Left) The low tilt case with $\theta=3 \mathrm{deg}$. (Middle) The moderate tilt case with $\theta=7 \mathrm{deg}$. (Right) The high tilt case with $\theta=15 \mathrm{deg}$.

(N)

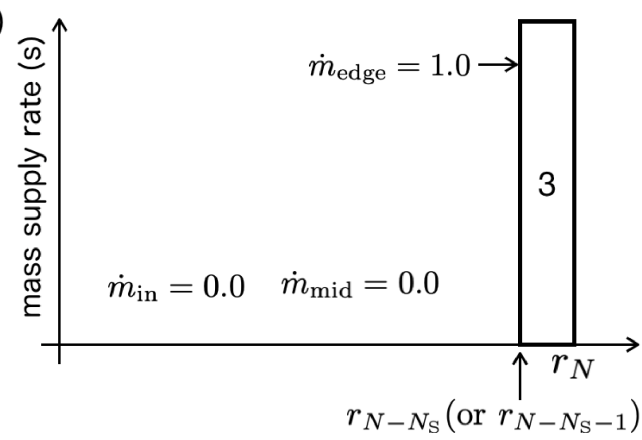

(B)

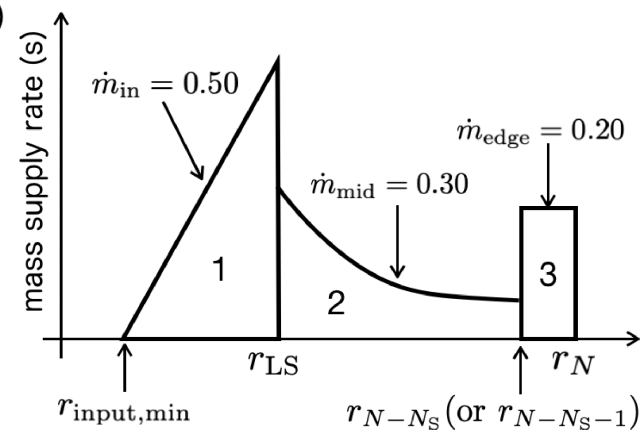

(A)

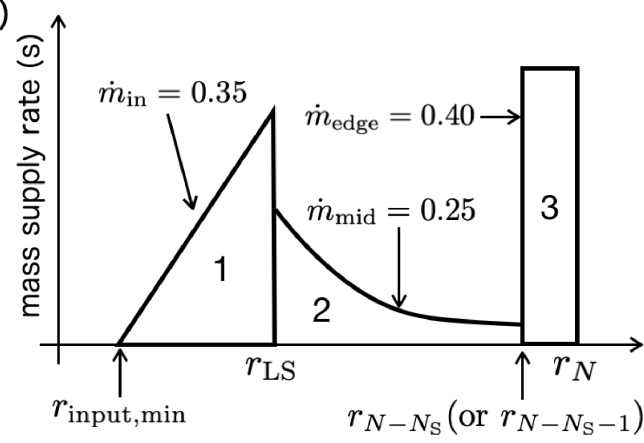

(C)

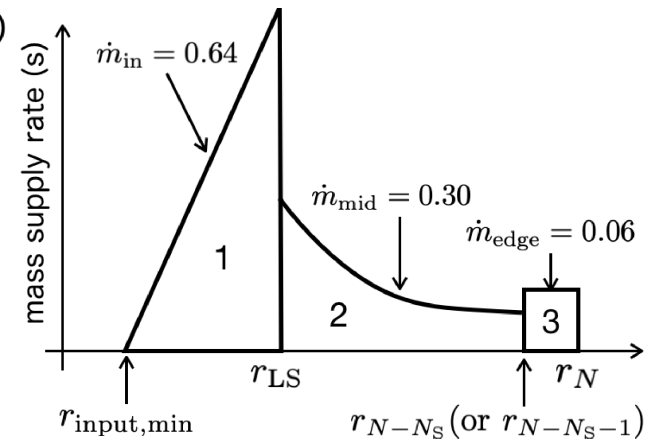

Fig. 8. Schematic pictures of mass input patterns that we use in our simulations on the basis of the results shown in Figure 7 . The regions 1,2 , and 3 are the annulus between $r_{\text {input,min }}$ and $r_{\mathrm{LS}}$, and that between $r_{\mathrm{LS}}$ and $r_{N-N_{\mathrm{S}}}\left(\right.$ or $\left.r_{N-N_{\mathrm{S}}-1}\right)$, and that between $r_{N-N_{\mathrm{S}}}\left(\right.$ or $\left.r_{N}-N_{\mathrm{S}}-1\right)$ and $r_{N}$, respectively. Pattern $(\mathrm{N})$ : The non-tilted standard case where the gas stream always enters the outer edge of the disk. Pattern (A): The low tilt case corresponding to the left panel of Figure 7, Pattern (B): The moderate tilt case corresponding to the middle panel of Figure 7, Pattern (C): The high tilt case corresponding to the right panel of Figure 7.

Appendix 3 for reference.

We have shown four mass input patterns including the nontilted, slightly-tilted, moderately-tilted, and highly-tilted cases in the previous subsection (see also Figure 8), and examine them with a constant mass transfer rate. The models and parameters that we deal with are summarized in Table 1. The mass input pattern and the mass transfer rate do not change with time within one model.

\section{Results of numerical simulations}

\subsection{Case of a non-tilted disk (Model N1)}

As mentioned in Sec. 3.1, the non-tilted standard case is a special case of our model with the zero tilt angle (see also Figure 8). It is very convenient to use this case as a test of our numerical code. We have first calculated this case with $\dot{M}_{\mathrm{tr}}=10^{16.75} \mathrm{~g} \mathrm{~s}^{-1}$ (i.e., Model N1) by our code. The resultant time evolution of the disk is shown in Figure 10. In calculating the $V$-band absolute magnitude, we consider the disk luminosity and the bright spot, and use the same method as that described in Dubus et al. (2018). We set the inclination angle to 


\begin{tabular}{|c||c|c|c|c|}
\hline models & mass input pattern & degree of tilt & $M_{\mathrm{tr}^{\dagger}}$ & figure(s) \\
\hline \hline $\mathrm{N} 1$ & $\mathrm{~N}$ & zero & $10^{16.75}$ & 10,11 \\
$\mathrm{~A} 1$ & $\mathrm{~A}$ & low & $10^{16.75}$ & 11 \\
$\mathrm{~B} 1$ & $\mathrm{~B}$ & moderate & $10^{16.75}$ & $11-14$ \\
$\mathrm{C} 1$ & $\mathrm{C}$ & high & $10^{16.75}$ & 11 \\
\hline $\mathrm{N} 2$ & $\mathrm{~N}$ & zero & $10^{17}$ & 15 \\
$\mathrm{~A} 2$ & $\mathrm{~A}$ & low & $10^{17}$ & 15 \\
$\mathrm{~B} 2$ & $\mathrm{~B}$ & moderate & $10^{17}$ & 15 \\
$\mathrm{C} 2$ & $\mathrm{C}$ & high & $10^{17}$ & 15 \\
\hline $\mathrm{N} 3$ & $\mathrm{~N}$ & zero & $10^{16.5}$ & 17 \\
$\mathrm{~A} 3$ & $\mathrm{~A}$ & low & $10^{16.5}$ & 17 \\
$\mathrm{~B} 3$ & $\mathrm{~B}$ & moderate & $10^{16.5}$ & 17 \\
$\mathrm{C} 3$ & $\mathrm{C}$ & high & $10^{16.5}$ & 17 \\
\hline $\mathrm{N} 4$ & $\mathrm{~N}$ & zero & $10^{17.25}$ & 18 \\
$\mathrm{~A} 4$ & $\mathrm{~A}$ & low & $10^{17.25}$ & 18 \\
$\mathrm{~B} 4$ & $\mathrm{~B}$ & moderate & $10^{17.25}$ & 18 \\
C4 & $\mathrm{C}$ & high & $10^{17.25}$ & 18 \\
\hline
\end{tabular}

${ }^{*}$ See Figure 8 for the definition of the mass input patterns.

${ }^{\dagger}$ In the unit of $\mathrm{g} \mathrm{s}^{-1}$.

Table 1. Summary of the models and parameter sets in our simulations.

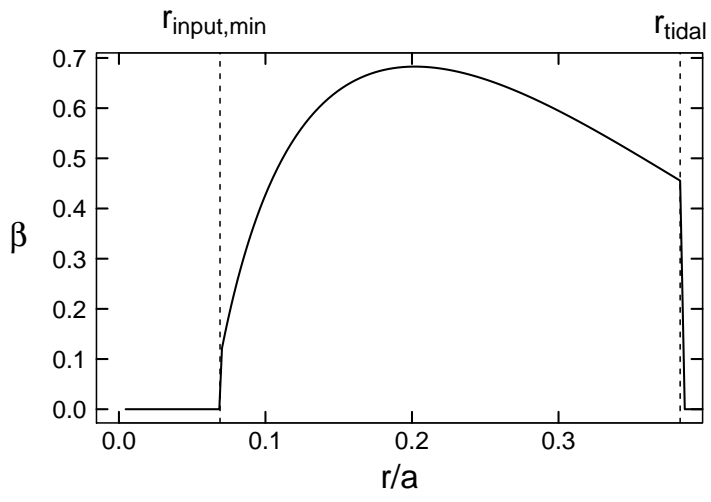

Fig. 9. Fraction of the thermally dissipated energy by the gas stream with respect to $G M_{1} / 2 r, \beta$, depending on the radius where the gas stream collides with the tilted disk. We estimate this value between $r_{\text {input,min }}$ and $r_{\text {tidal }}$.

be $45 \mathrm{deg}$. Here the inclination angle is not the inclination of the binary system but the inclination of the disk to the observer. We assume that the disk emission is multi-color blackbody, and that the bright spot emits single-temperature blackbody. We assume the size of the bright spot is $2 \%$ of the disk, and its luminosity is simply approximated to be $0.25 G M \dot{M}_{\mathrm{tr}} / r_{N}$.

As seen in Figure 10, our results reproduce outside-in outbursts which are typical of dwarf-nova outbursts. The variations of disk luminosity, disk radius, disk mass, and disk angular momentum are similar to those in the past simulation works (e.g., Ichikawa, Osaki 1992; Hameury et al. 2000). We see that the resultant light variations regularly repeat long and short outbursts. This is not common in all DNe, but is observed in SS Cyg at least in some epochs (e.g., during JD 2450100-2450450 in Fig. 1 in McGowan et al. (2004)). The disk radius suddenly increases at the onset of outbursts because of a sudden increase in the angular momentum transport from the inner disk to the outer disk, while the disk slowly shrinks after the luminosity maximum because of addition of mass having low specific angular momentum at the outer edge in the cool disk. These reproduce the typical observational disk-radius variations (e.g., Smak 1984). The disk radius becomes constant around the luminosity maxima because of the tidal truncation (see also Sec. 3.5 and Appendix 1).

The bottom panel of Figure 10 represents the time variations of the nodal precession rate $\left(\nu_{\mathrm{nSH}}\right)$ of a tilted rigid disk. If the tilt angle of the disk is very low, the gas stream would almost always enter around the disk edge. Then the time evolution of the disk would be almost the same one as that in the non-tilted case. If we calculate the variations of $\nu_{\mathrm{nSH}}$ in this case, they are good approximations of those in the very low tilt case. If we do not consider the time variation of radial mass distribution in the disk, $\nu_{\mathrm{nSH}}$ tracks the disk-radius variation (the dashed line); however, more realistic variations in $\nu_{\mathrm{nSH}}$ reflect the timeevolving mass distribution (the solid line). We give the detailed explanation in Appendix 2. 


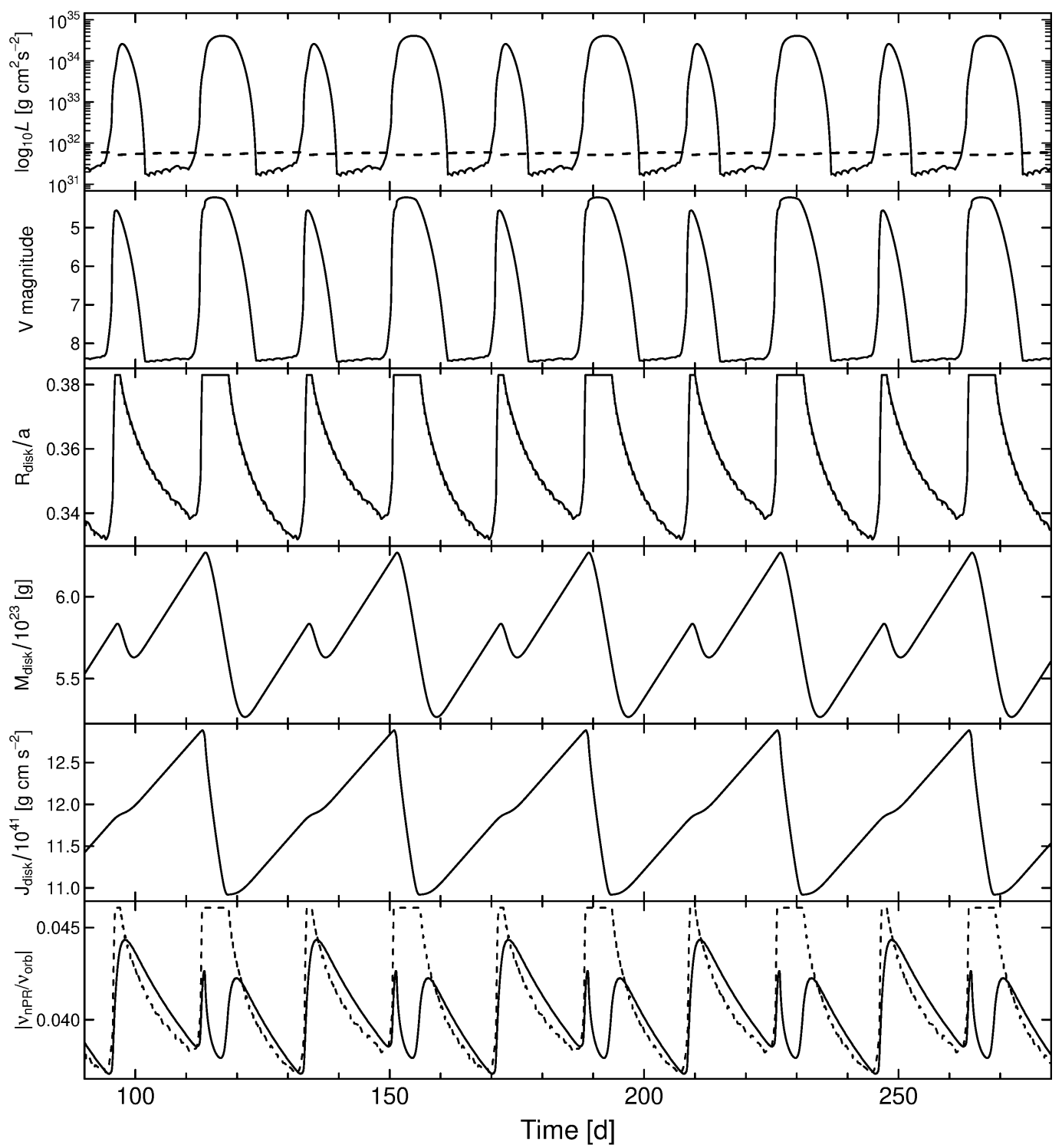

Fig. 10. Time evolution of the non-tilted accretion disk in the case of $\dot{M}_{\mathrm{tr}}=10^{16.75} \mathrm{~g} \mathrm{~s}^{-1}$ (Model N1 in Table 1). From top to bottom: the luminosity of the disk, the absolute $V$-band magnitude, the disk radius in the unit of the binary separation, the total disk mass, the total angular momentum, and the absolute value of the normalized nodal precession rate of the disk. The dashed line at the top panel represents the luminosity of the bright spot $\left(=0.25 G M \dot{M}_{\mathrm{tr}} / r_{N}\right)$. The observed luminosity in quiescence is expected not to be lower than this line. The dashed line in the bottom panel represents the nodal precession rate calculated by equation (A18) with $\eta=1.17$.

\subsection{How do the light variations change by the disk tilt ?}

Now we are ready to investigate how the light curves change from those in the non-tilted standard case when the disk is tilted. We show the resultant $V$-band light curves of the simulations with four mass input patterns in the case of the same mass transfer rate as that in the previous subsection $\dot{M}_{\mathrm{tr}}=10^{16.75} \mathrm{~g} \mathrm{~s}^{-1}$ (Models N1, A1, B1, and C1) in Figure 11. Here we show later parts of simulations in which the effects of the initial condition have already faded, and some arbitrary offsets of time are made for visibility. The luminosity of the bright spot is in- cluded in the light curves in the tilted cases (Models A1, B1, and $\mathrm{C} 1$ ) as in the non-tilted case (Model N1), and it is assumed to be $0.25 G M \dot{M}_{\mathrm{tr}} / r_{\mathrm{LS}}$ for simplicity, since the gas stream from the secondary arrives between $r_{\text {input,min }}$ and $r_{N}$. Since the gas stream reaches deeper in the potential well in the tilted cases as compared with that in the non-tilted case, the level of minima in $V$-band luminosity is thus higher in the tilted disk than that in the non-tilted disk (see the top and 2nd panels of Figure 11).

In Figure 11, we can see that diverse light variations appear in the tilted disk depending on the mass input pattern, for the same mass transfer rate. As described in the preceding subsec- 


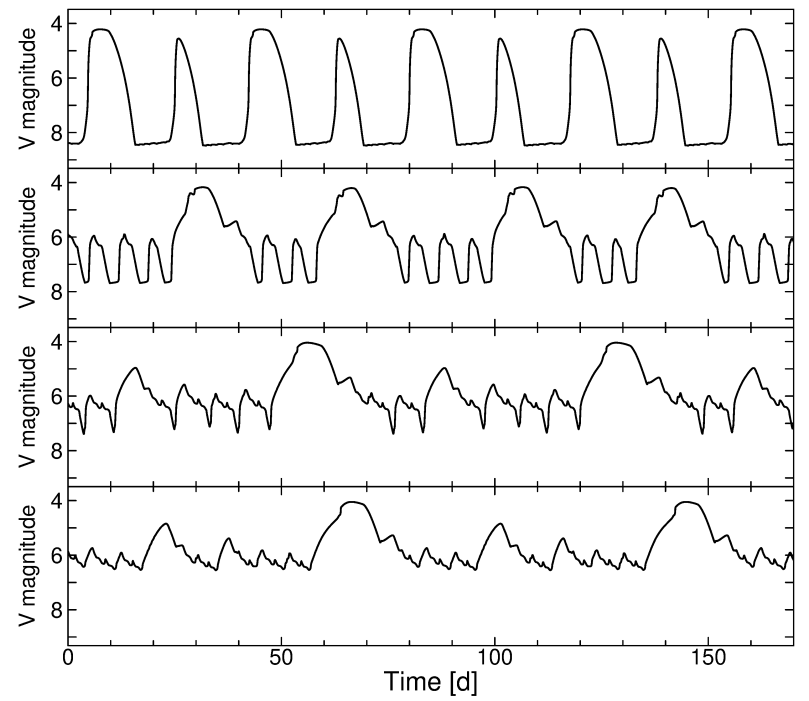

Fig. 11. Time evolution of the $V$-band magnitude of the tilted accretion disk in the case of $\dot{M}_{\mathrm{tr}}=10^{16.75} \mathrm{~g} \mathrm{~s}^{-1}$. The contribution of the bright spot is included in our simulations. From top to bottom: the non-tilted standard case (Model N1), the low tilt case with mass input pattern (A) (Model A1), the moderate tilt case with mass input pattern (B) (Model B1), and the high tilt case with mass input pattern (C) (Model C1).

tion, the disk alternately experiences long and short outbursts in the non-tilted disk (the top panel). In Model A1, the disk alternates between a large outburst and a few small outbursts (the 2nd panel). In Model B1, large outbursts sandwich midbrightness interval with repetitive dips (the 3 rd panel). In Model $\mathrm{C} 1$, we see repetition of oscillations and brightening (the bottom panel). The interval between brightening becomes longer and the amplitude of outbursts becomes smaller as the tilt becomes larger. In the tilted cases, we always see slow rise at the onset of outbursts (or brightening), which represent inside-out outbursts (Mineshige, Osaki 1985). Since the fraction of gas stream arriving in the inner part of the disk becomes larger in the tilted disk, the outbursts are easily triggered in the inner disk.

The light curve in Model B1 shown in the 3rd panel of Figure 11 is most interesting, since it shows a large outburst and a middle level brightness oscillation accompanied with a dip. We therefore examine the results in this case in detail.

\subsection{Time evolution of the disk in Model B1}

We show the time-dependent properties of an accretion disk in Model B1 in Figure 12. The interval during 221-293 days in this figure corresponds to one cycle, consisting of a large outburst, and oscillatory light variations in mid-brightness interval occasionally accompanied with dips. In the bottom panel of Figure 12, we also estimate the variations of the nodal precession rates as we do in Model N1. Their time variations are dominated by the time evolution in radial mass distribution in the disk (the solid line) rather than the disk-radius variations (the dashed line). We explain in detail this behavior in Appendix 2.

To help understanding this behavior, we show two more figures, Figures 13 and 14. In Figure 13, we show three snapshots of the distributions of temperature (the left column) and surface density (the right column), where times shown as three circles in Figure 12 (numbered as 1, 2, and 3 in its top panel) correspond to those at a large outburst, the middle-level brightness interval, and a dip, respectively. The dashed line in each panel in the left column represents the minimum temperature for achieving the hot state. The dashed line in the 2 nd and 3rd panels in the right column represents the maximum surface density for keeping the entire disk cool.

Figure 14 shows time variations of temperature in one cycle at three representative radii of the outer, the middle, and the inner parts of the disk $\left(\log _{10} r=10.43,10.16\right.$, and 9.75) from the top to the bottom, respectively. Although the light curve itself is very complicated in one cycle, the time variations of the total angular momentum in the disk, $J_{\text {disk }}$, in the 5 th panel of Figure 12, show simply slow monotonic increase and then rapid monotonic decrease after its maximum in one cycle. We thus use $J_{\text {disk }}$ as a good indicator of the phase of one cycle. The evolutionary tracks for two cycles in Figure 14 completely overlap and the arrows in the top panel represent the direction of time evolution.

We can understand roughly this behavior by dividing the disk into three parts, the inner, the middle, and the outer parts. The inner part spends most of the time in the hot state (see, the bottom panel of Figure 14), and the outer part stays in the cool state for most of the time during one cycle (its top panel), and in the middle part between the hot inner part and the cool outer part, transition waves (i.e., heating waves and cooling waves) frequently go back and forth there (its middle panel). Thus different regions of the disk behave differently.

At the beginning of one cycle (at brightening), the whole disk stays in the hot quasi-steady state (see the 1st panel of the left column in Figure 13). After the end of a large outburst, the cooling wave starting from the outer edge propagates inward and it transforms the outer part of the disk into the cool state. While the outer part gradually accumulates mass in the cool state (see, the 4th panel of Figure 12, and the 2nd and 3rd panels of the right column in Figure 13), the inner part usually stays in the hot state (see the 2nd panel of the left column of Figure 13), and makes an intermediate-brightness state. Cooling and heating waves alternately develop in the middle region (see the middle panel of Figure 14), and it causes oscillatory variations in the disk luminosity. Occasionally a cooling wave propagating to the inner part causes luminosity drop, i.e., 6 dips in the light curve correspond to 6 drops in temperature in the inner disk per cycle (see the bottom panel of Figure 14 and the 3rd panels of Figure 13). Finally, a heating wave developed at the middle part propagates outward all the way and reaches the outer edge 


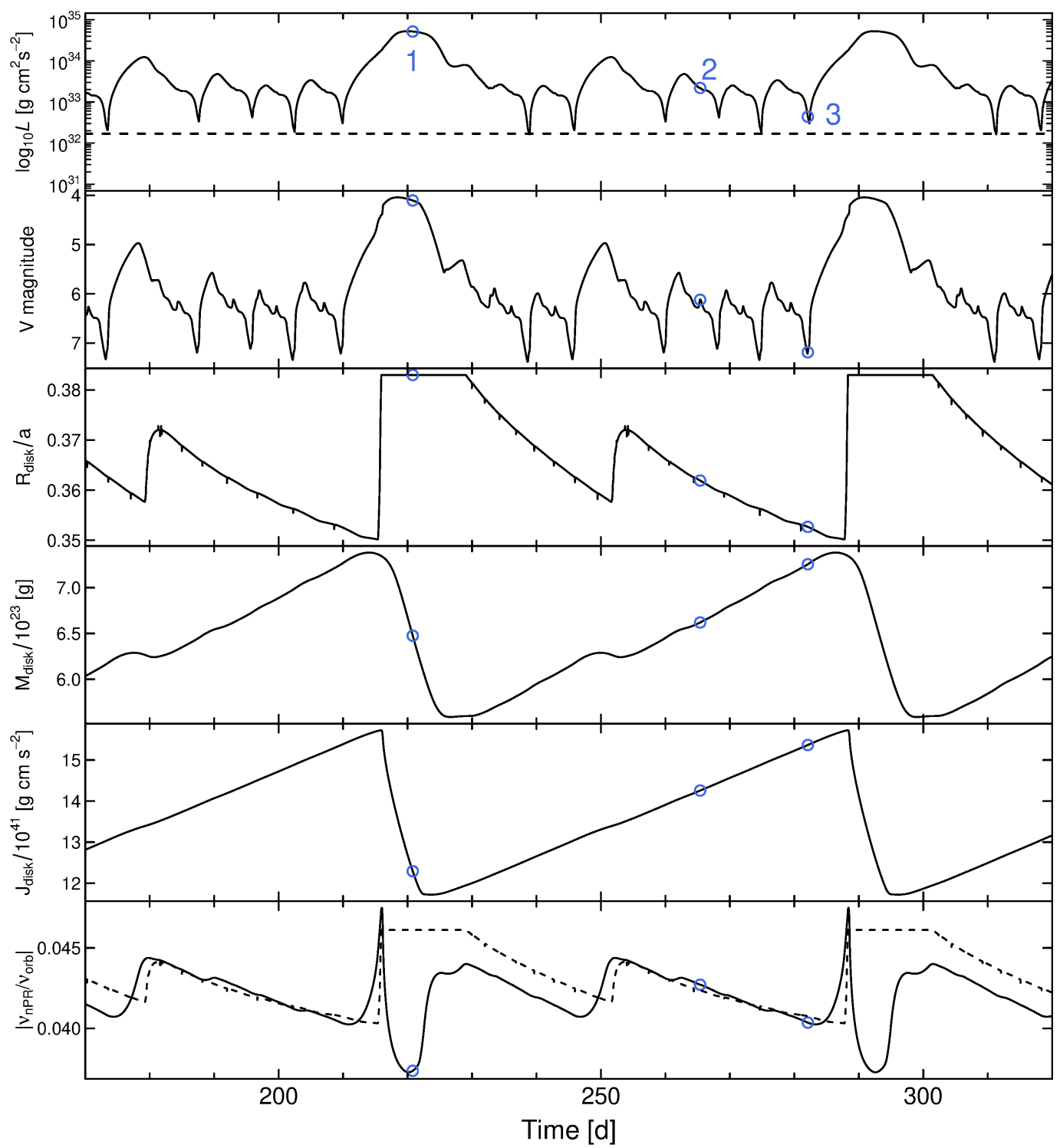

Fig. 12. Time evolution of the tilted accretion disk in the case of $\dot{M}_{\mathrm{tr}}=10^{16.75} \mathrm{~g} \mathrm{~s}^{-1}$ with mass input pattern (B) displayed in the lower-left panel of Figure 8 (Model B1 in Table 1). From top to bottom: the same ones as in Figure 10. The circles correspond to the time picked up at each panel in each column of Figure 13. We assume that the luminosity of the bright spot is approximately represented as $0.25 G M \dot{M}_{\mathrm{tr}} / r_{\mathrm{LS}}$. Also, we use 1.18 as $\eta$ value in calculating the dashed line in the bottom panel.

of the disk, and it turns the entire disk into the hot state (see the top panels of Figure 13). At that time, brightening is regenerated. The drain of a large amount of mass onto the central white dwarf during the large inside-out outburst returns the disk to the starting point of one cycle. The time necessary to accumulate a large amount of mass in the outer disk determines the duration of one cycle. The disk thus experiences a new type of accretion cycle if the gas stream from the secondary penetrates to the inner disk.

\subsection{Brief explanations for the light variations in Model A1 and Model C1}

We explore the light curves in the other tilt cases for $\dot{M}_{\mathrm{tr}}=$ $10^{16.75} \mathrm{~g} \mathrm{~s}^{-1}$ at first on the basis of our investigation in Sec. 5.3. In Model A1, there are clear quiescent states between outbursts and the more frequent large outbursts as compared with those in Model B1 (see, the 2nd and 3rd panels of Figure 11). This is because the inner disk often drops to the cool state and keeps cool for longer time due to the smaller mass input to the inner disk, and because the outer disk often enters into the hot state due to the larger mass input to the outer edge of the disk. On the other hand, in Model C1, dips seen in Model B1 disappear, because 

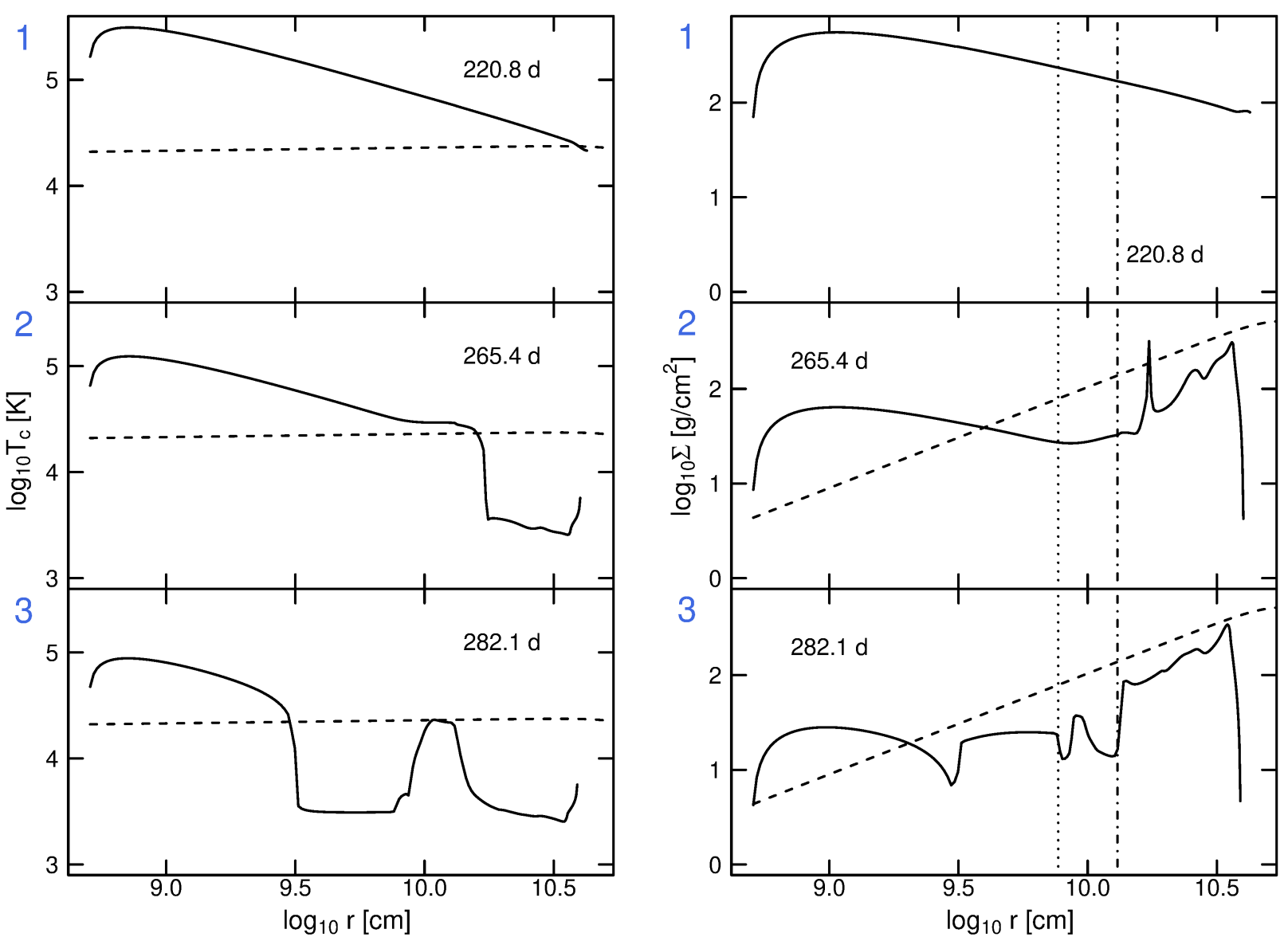

Fig. 13. A part of time evolution of the temperature (left) and the surface density (right) of the disk in the case of Figure 12. The corresponding time (date) is written in each panel. They are also marked at the top panel of Figure 12. The dashed line in the left panel represents the minima of temperature $\left(T_{\mathrm{hot}}, \mathrm{min}\right)$ for achieving the hot state as calculated in equation (38) in Ichikawa, Osaki (1992). The dashed line in the right panel represents the maxima of surface density $\left(\Sigma_{\text {cool,max }}\right)$ for keeping the disk cool as calculated in equation (35) in Ichikawa, Osaki (1992). The dot line and dash-dotted line in the right plane represent $r_{\text {input, min }}$ and $r_{\mathrm{LS}}$, respectively.

the inner disk always stays in the hot state due to the sufficient mass supply inside $r_{\mathrm{LS}}$ (see the bottom panel of Figure 11). The outer disk stays longer in the cool state because of reduced mass supply to it, and hence, the duration of one cycle becomes longer as compared with that in Model B1.

\subsection{Brief explanations for the light variations in the case of other mass transfer rates}

We next examine the light variations for different mass transfer rates. The resultant $V$-band light curves for 4 different mass input patterns with $\dot{M}_{\mathrm{tr}}=10^{17} \mathrm{~g} \mathrm{~s}^{-1}$ (Models N2, A2, B2, and $\mathrm{C} 2$ ) in Figure 15 . We see the quiescent state completely disappears in the tilt cases (Models A2, B2, and C2). Under this high mass transfer rate, the mass input rate to the inner disk is high enough to keep it hot even in Model A2. Small-amplitude brightening is repeated in Models A2 and B2, since the interval between brightening becomes shorter and the mid-brightness interval disappears because of the large amount of mass supply to the outer disk edge. The behavior in Model C2 is similar to that in Model C1. We also explain the other cases in Appendix 3 .

\subsection{Test simulations with another set of binary parameters}

We also have checked whether our main results are sensitive for the binary parameters or not, by performing some simulations with the binary parameters of KIC 9406652 (Gies et al. 2013). The details of the parameter set and the method are described in Appendix 4. We have tried simulations of the non-tilted standard case and the three tilted cases in the case of $\dot{M}_{\mathrm{tr}}=10^{16.9} \mathrm{~g} \mathrm{~s}^{-1}$ by using the same mass input patterns as those shown in Figure 8. The resultant $V$-band light curves are exhibited in Figure 16. The results are similar to those in Figure 11 , and the major features in the simulations with the binary parameters of U Gem: frequent outside-in outbursts in the nontilted case, repetition of small inside-out outbursts and a large 


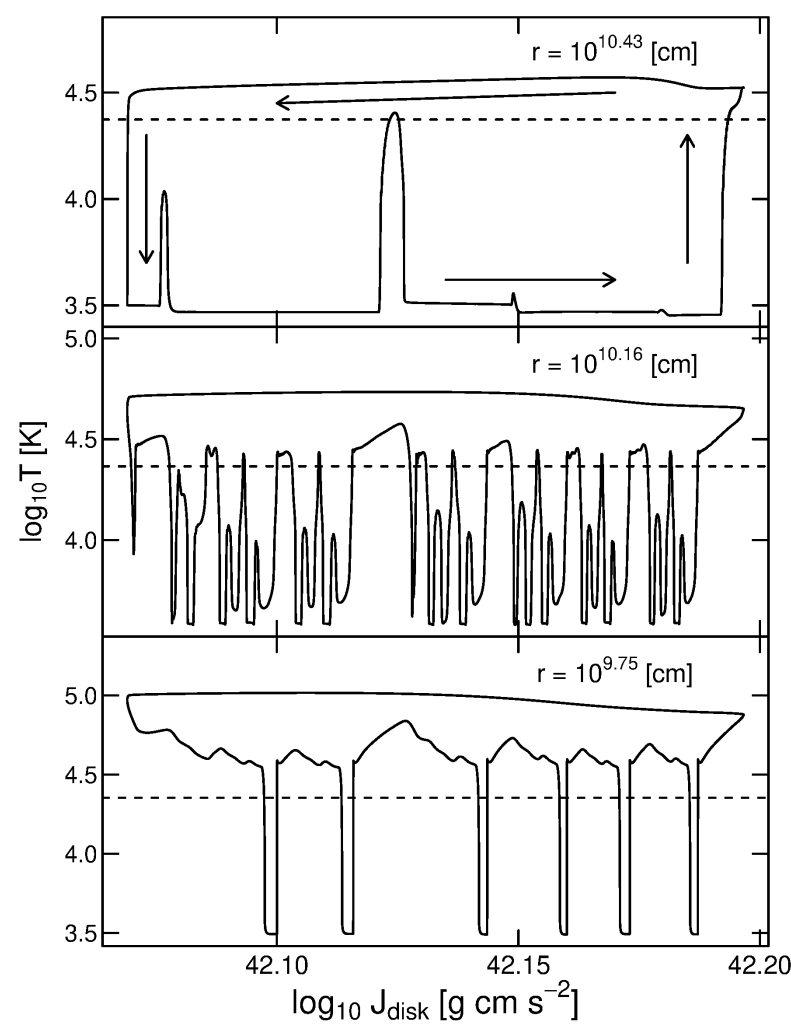

Fig. 14. $T$ - $J_{\text {disk }}$ planes at three representative points in the disk, i.e, $\log _{10} r=10.43,10.16$, and 9.75 , corresponding to the outer, the middle, and the inner parts of the disk, respectively, during 170-320 days. The arrows in the top panel represent the direction of time evolution. The dashed line denotes $T_{\mathrm{hot}, \mathrm{min}}$ defined in Figure 13.

inside-out outburst in the low tilt case, repetition of oscillatory state with dips terminated by brightening in the moderate tilt case, and that without dips in the high tilt case are reproduced. We thus have confirmed a new accretion cycle also occurs if the system has a tilted disk and a relatively high mass transfer rate.

\section{Discussion}

\subsection{Comparison of our simulations with observations}

As described in Sec. 2, there are the two essential features of the light variations in IW And-type DNe; cyclic light variations with a characteristic pattern, which we call the IW And-type phenomenon, and a wide variety in the long-term light curves even within one object. Our simulations for the tilted disk give the following main results with respect to these features of IW And stars.

- Some of our simulations of the tilted disk can produce light curves reminiscent of the IW And-type phenomenon: regular repetition of the mid-brightness interval with oscillations and sometimes dips followed by an outburst (brightening) (see, the third and the bottom panels of Figure 11, the bottom panel

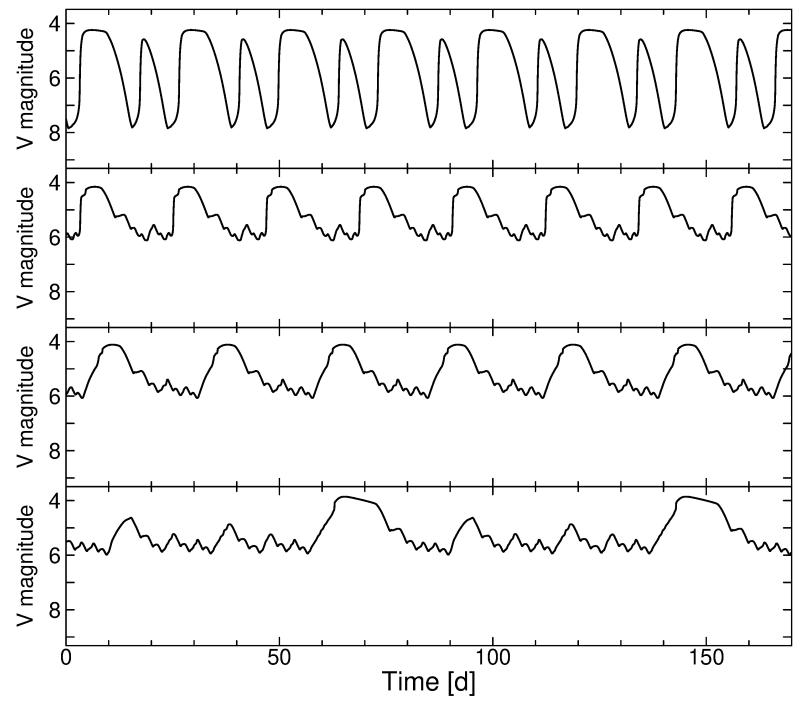

Fig. 15. Same as Figure 11 but for $\dot{M}_{\mathrm{tr}}=10^{17} \mathrm{~g} \mathrm{~s}^{-1}$ (Models N2, A2, B2, and C2).

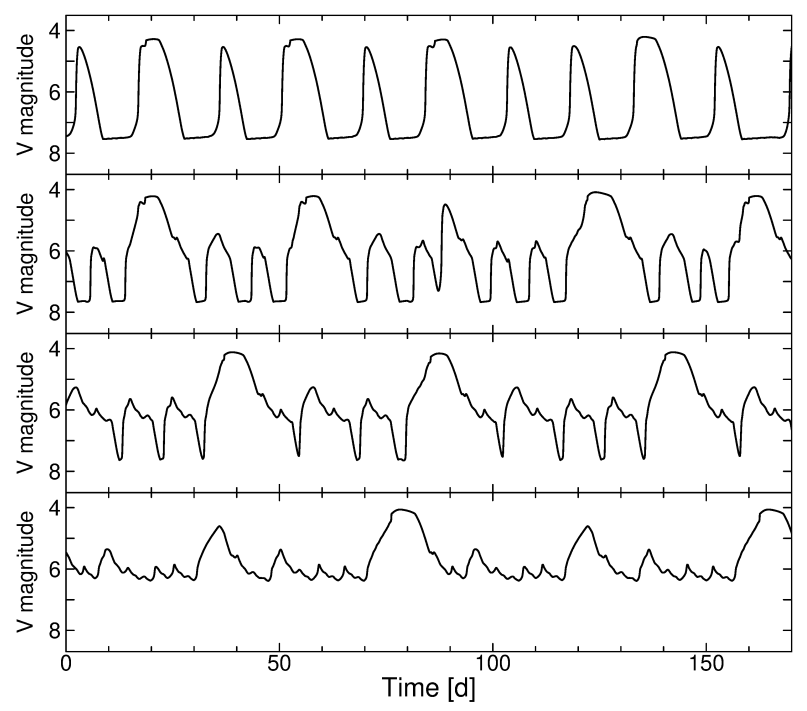

Fig. 16. Same as Figure 11 but for $\dot{M}_{\mathrm{tr}}=10^{16.9} \mathrm{~g} \mathrm{~s}^{-1}$ and with the binary parameters of KIC 9406652.

of Figure 15, and the third and the bottom panel of Figure 16). This is produced because the inner, the middle, and the outer regions behave differently in the tilted disk where the gas stream reaches not only the outer disk edge but also the inner disk. The inner disk mostly stays in the hot state and it helps to sustain the mid-brightness interval. The outer disk spends long time in the cool state, and once it accumulates enough mass, an inside-out outburst occurs which terminates the mid-brightness interval. Cooling and heating waves frequently go back and forth in the middle region between the inner hot disk and the outer cool disk, which produces oscillatory variations in the mid-brightness interval. A cooling 
wave occasionally reaches the inner disk, and a dip (a quiescence) then appears in the light curve. These phenomena combined can produce a kind of new accretion cycle in tilted disks as suggested in Kato (2019).

- Our simulations have demonstrated that the thermal instability working on the tilted disk can produce a wide variety in light curves depending on mass input patterns, i.e., the tilt angle and/or the disk geometry, even with constant mass transfer rates (see Figures 11, 15, and 16). That is, different mass input patterns make different lengths of intervals between brightening and different excursion time to the cool state (i.e., the difference between clear quiescence, a short dip, and no quiescence in the mid-brightness interval). For example, the systems can alternate between dwarf-nova outbursts, repetition of intermediate-brightness state terminated by brightening, which resembles to the IW And-type phenomenon, and repetitive brightening similar to heartbeattype oscillations observed in HO Pup (see the lower panel of Figure 2 and Figure 15) if the mass transfer rate is relatively high and if the mass input pattern varies on long timescales.

On the other hand, we have found it difficult to explain by our model the details of the observational IW And-type light variations. For example, luminosity dips sometimes appear just after brightening in observations (see also Figures 1 and 2), but in our simulations, dips appear during the mid-brightness interval instead of after brightening (see the 3rd panel of Figure 11). Also, the amplitudes of brightening are typically less than 1 mag in observations, but they are $\sim 1$-mag larger in our simulations. Although oscillations in quasi-standstills are sometimes moderate in the observational light curves (see also Figure 1), our simulations always show large-amplitude oscillations in the mid-brightness interval. Z Cam-type standstills are sometimes observed in long-term light curves as well (see the upper panel of Figure 2), but our simulations do not reproduce them. This is because the inner hot and the outer cool disks coexist when the mid-brightness interval is generated by our models. The luminosity never settles down in this case, since cooling and heating waves are always triggered between the hot and the cool regions. Since we have performed our numerical simulations with a certain set of assumptions, and since we have not explored other possible assumptions, it is not expected to reproduce all of observational details of IW And-type stars in this work. Other possible assumptions to be explored are such as those, for instance, more realistic thermal equilibrium curve instead of the simple ' $\mathrm{S}$ '-shaped thermal equilibrium curve, other combinations of the viscosity parameters, $\alpha_{\text {hot }}$ and $\alpha_{\text {cold }}$, and other forms of mass input patterns, and so on. Those attempts are beyond the scope of this paper.

\subsection{Would IW And-type dwarf novae have tilted disks?}

Our simulations suggest light variations similar to the IW Andtype phenomenon appear in tilted disks with relatively high mass transfer rates which are close to those of Z Cam-type DNe (see, the third and the bottom panels of Figure 11, and the bottom panel of Figure 15). Although the mechanisms for inducing tilted or warped structures are controversial, Montgomery, Martin (2010) proposed that lift may force a disk to be tilted. The lift is the same force as that exerted on the surface area of the wings of airplanes. They showed the tilt by lift happens more easily in the objects having higher mass transfer rates. Their and our results are consistent with the recent observations in that the IW And-type phenomenon is prevalent in $\mathrm{Z}$ Cam-type DNe (e.g., Kato 2019). Therefore the disk tilt is regarded as a promising assumption to explain the IW And-type phenomenon, and we could naturally interpret that SS Cyg stars having lower mass transfer rates never show this kind of phenomenon.

As displayed in the bottom panels of Figure 10 and 12, we can now calculate the frequency variations of negative superhumps (see also Appendix 2). The comparison between our calculations and observations might give another evidence of the disk tilt and our model. Since the time variations in the precession rate reflect the changes in mass distribution within the disk, we can also discuss the mass distribution in the disk through the variations in negative-superhump periods with high time-cadence light curves. We try these works in our next paper.

\subsection{Possibility of formation of a gap in the disk}

Let us come back to a problem of the mass input pattern discussed in Sec. 4.1, particularly about a triangular profile in the region 1 (see also Figure 8), and we now discuss why we have used such a profile in this work. Initially we started our simulations by adopting a step function in region 1 for the source function, $s(r)$, in the same way as in region 3. However, we had to stop our computation because we encountered some numerical difficulty around $r_{\text {input,min }}$ when the inner part of the disk becomes in the cool state. What happened was that a vacuum (or a gap in the disk) seemed to be formed around $r_{\text {input,min }}$, although our code could not treat such a hole.

If the inner disk becomes in the cool state, the disk matter hardly moves there because of the low viscosity. Since the inner part of the disk below $r_{\text {input,min }}$ has no mass supply from the secondary star, the disk matter sits in its place in such a case. On the other hand, the disk matter between $r_{\text {input,min }}$ and $r_{\mathrm{LS}}$ receives mass from the secondary star with the specific angular momentum $\left(\sqrt{G M r_{\mathrm{LS}}}\right)$ larger than that of the disk matter, and is forced to move rapidly towards the Lubow-Shu radius. This is because the third term of the right hand side in equation (8) 
takes a large negative value which dominates over the viscous diffusion term (i.e., its first term). In this case, the disk may split into the two parts, the inner cool disk and the outer disk having an inner boundary around the Lubow-Shu radius.

To avoid such a difficulty, we have used such a mass input profile in the region 1 where the source term, $s(r)$, tapers down to zero at $r_{\text {input,min. }}$. In our problem with our interest, the cool state in the inner part of the disk is rather short and we have been able to avoid that numerical difficulty by this treatment. In fact, even in our treatment we can see such a tendency of thinning disk matter just above $r_{\text {input,min }}$ as seen in the third panel of the right column in Figure 13 when the inner disk stays in a cool state. Although full 3-dimensional hydrodynamic simulations may be necessary to confirm this, the formation of a gap in the disk seems very likely to occur if the gas stream from the secondary penetrates deeply in the cool disk. We need to treat such a case in future.

\section{Summary}

We have performed numerical simulations of the disk instability of dwarf novae in the case of tilted disks by taking into account the various mass supply patterns when the gas stream penetrates deeply in the disk under some simplifying assumptions. We have found that a new kind of accretion cycle can occur in tilted accretion disks as suggested in Kato (2019). This is made possible because the different parts of the disk (i.e., the inner, the middle, and the outer parts of the disk) can stay in different thermal states in tilted disks. The inner disk mostly stays in the hot state while the outer disk spends most of time as the cool state but the latter eventually makes an outburst when enough mass is accumulated there. As a result, alternating mid-brightness interval (repeating small outbursts) and brightening (a large outburst) are reproduced. The oscillatory light variations in the mid-brightness interval are inevitable because cooling and heating waves are always triggered in the middle part between the hot inner disk and the cool outer disk.

By this cyclic accretion, light variations reminiscent of the IW And-type phenomenon appear when the mass transfer rate is relatively high. The thermal instability in the tilted disk would be a plausible model for explaining the most characteristic feature of the IW And-type phenomenon. Furthermore, we have found that a quite different variety in light curve patterns can be produced depending on the mass supply pattern in tilted disks even within a given mass transfer rate, which could be a key for understanding a wide variety in the long-term light curves of IW And stars. Successful reproduction of the essential features of IW And-type light curves is in favor of by the thermal-viscous instability in the tilted disk, although we could not reproduce the details such as the mid-brightness interval with low-amplitude oscillations and Z Cam-type standstills by our model. This point is left as a future issue.

\section{Acknowledgements}

This work was financially supported in part by the Grantin-Aid for JSPS Fellows for young researchers (MK) and by JSPS Grant-in-Aid for Scientific Research (C) (17K0583, SM). We are grateful to Hiromoto Shibahashi. We are also grateful to the participants of "IM Eri campaign" led by the VSNET Collaboration (Osaka Kyoiku Univ. team, Tonny Vanmunster, Franz-Josef Hambsch, Hiroshi Itoh, Crimean Astrophys. Observatory team, Berto Monard, Kyoto Univ. team, and Shawn Dvorak). We would like to express our appreciation to anonymous referees.

\section{Appendix. 1. Details of finite-difference scheme}

As mentioned in the main texts, the finite-difference scheme used in this paper is the same as that described in Ichikawa, Osaki (1992). We divide the accretion disk into $N$ concentric annuli, and define the interface between $i$-th annulus and $(i+1)$ th annulus as $r_{i}$, and the center of $i$-th annulus as $r_{i-1 / 2}$ as described in Sec. 3.5. We give $\Sigma$ and $T_{\mathrm{c}}$ at the center of each annulus and these variables are expressed with the half-integer subscript $i-1 / 2$, while the mass accretion rate, $\dot{M}$, is defined at the interface of each annulus with the integer subscript $i$. The inner boundary of the disk is now given by $r_{0}$ and the outer boundary is given by $r_{N}$. In this study we choose the inner boundary at the surface of the primary white dwarf.

As for the time integration, we adopt a hybrid method of explicit integration for mass conservation and implicit integration for the energy equation. The mass of the $i$-th annulus, $\Delta M_{i-1 / 2}$ is then given by $\Delta M_{i-1 / 2}=\pi\left(r_{i}^{2}-r_{i-1}^{2}\right) \Sigma_{i-1 / 2}$ and the equation of mass conservation (i.e., equation (2)) is now written in our finite-difference scheme as

$$
\begin{gathered}
\Delta M_{i-1 / 2}^{\text {new }}=\Delta M_{i-1 / 2}+\left(\dot{M}_{i}-\dot{M}_{i-1}\right) d t+\dot{M}_{\mathrm{S}, i-1 / 2} d t,(\mathrm{~A} 1) \\
(\text { for } i=1,2,3, \ldots, N),
\end{gathered}
$$

where $d t$ is the time step, and $M_{\mathrm{S}, i-1 / 2}=\int_{r_{i-1}}^{r_{i}} s(r) d r$ is mass supply rate to the $i$-th annulus. The variable with the subscript "new" means that at the new time step. On the other hand, as for the conservation of angular momentum, we use equation (8). By integrating equation (8) over $r_{i-1 / 2}$ and $r_{i+1 / 2}$, we obtain

$$
\begin{gathered}
\dot{M}_{i}\left(h_{i+1 / 2}-h_{i-1 / 2}\right)=\left(2 \pi r^{2} W\right)_{i+1 / 2}-\left(2 \pi r^{2} W\right)_{i-1 / 2}(\mathrm{~A} 2) \\
+\left[D+\left(h-h_{\mathrm{LS}}\right) s\right]_{i}\left(r_{i+1 / 2}-r_{i-1 / 2}\right) \\
(\text { for } i=0,1,2, \ldots, N-1),
\end{gathered}
$$

where the last term is defined in equation (10) of Ichikawa, Osaki (1992). The finite difference equation for the energy equation is written as follows: 


$$
\begin{array}{r}
C_{\mathrm{P}, i-1 / 2}^{\text {new }}\left[\Delta M_{i-1 / 2}^{\text {new }} T_{\mathrm{c}, i-1 / 2}^{\text {new }}-\Delta M_{i-1 / 2} T_{\mathrm{c}, i-1 / 2}\right] \\
C_{\mathrm{P}, i-1 / 2}^{\text {new }}\left[\left(\dot{M} T_{\mathrm{c}}\right)_{i}-\left(\dot{M} T_{\mathrm{c}}\right)_{i-1}+\dot{M}_{\mathrm{S}, i-1 / 2} T_{\mathrm{c}, i-1 / 2}^{\text {new }}\right] d t+ \\
\frac{4 \pi C_{\mathrm{P}, i-1 / 2}^{\text {new }} W_{i-1 / 2}^{\text {new }}}{\Omega_{i-1 / 2}}\left[r_{i} \frac{T_{\mathrm{c}, i+1 / 2}-T_{\mathrm{c}, i-1 / 2}}{r_{i+1 / 2}-r_{i-1 / 2}}-\right. \\
\left.r_{i-1} \frac{T_{\mathrm{c}, i-1 / 2}-T_{\mathrm{c}, i-3 / 2}}{r_{i-1 / 2}-r_{i-3 / 2}}\right] d t+ \\
\pi\left(r_{i}^{2}-r_{i-1}^{2}\right)\left(Q^{+}-Q^{-}\right)_{i-1 / 2}^{\text {new }} d t \\
(\text { for } i=1,2,3, \ldots, N),
\end{array}
$$

where $T_{\mathrm{c}}$ on the interfaces of annuli is chosen as that at the center of annuli at the upstream side of flow.

The most important point in this scheme is that the width of the outermost annulus is variable, while those of other annuli are fixed. Some special treatment is necessary to handle the outermost annulus and in our formulation we vary $r_{N}$ at each time step in such a way to conserve the total angular momentum of the disk, and its detailed description is given in Ichikawa, Osaki (1992) and that paper should be consulted (see, their equation (15)). The number of annuli $N$ is also variable as a mesh is either added or deleted if the width of the outermost annulus, $\Delta r_{N}$, exceeds some pre-specified size or shrinks below another pre-specified size when the disk expands or contracts. The way for increasing or decreasing the number of meshes is described in Sec. 2.3 of Ichikawa, Osaki (1992).

As for the inner boundary condition, we adopt $r_{-1 / 2}=r_{0}$ and $W_{-1 / 2}=0$, which means stress free at the inner edge of the disk. Here the mass accretion rate to the white dwarf, $\dot{M}_{0}$, is approximately calculated from equation (A2) as follows:

$\dot{M}_{0}=\left(2 \pi r^{2} W\right)_{1 / 2} /\left(h_{1 / 2}-h_{0}\right)$,

where we assume the tidal torque is minute at the innermost annulus, and $s_{1 / 2}=0$.

As for the outer boundary condition, we adopt $W_{N}=0$ and $\dot{M}_{N}=0$, which means no material outside the disk and that the mass does not escape from the outer disk edge. In this formulation, the outer disk absorbs the angular momentum transferred from the inside by its expansion when the outer disk enters the hot state, having high viscosity. When the disk reaches the tidal truncation radius $r_{\text {tidal }}$ and tries to expand beyond it, we fix the disk radius at that radius by removing the extra angular momentum from the disk. The extra angular momentum to be removed is calculated by using equation (15) in Ichikawa, Osaki (1992).

The total mass and total angular momentum of the disk are now written as

$$
\begin{aligned}
M_{\text {disk }} & =\int_{r_{0}}^{r_{N}} 2 \pi r \sum d r=\Sigma_{i=1}^{N} \Delta M_{i-1 / 2}, \\
J_{\text {disk }} & =\int_{r_{0}}^{r_{N}} 2 \pi r \Sigma h d r=\Sigma_{i=1}^{N} h_{i-1 / 2} \Delta M_{i-1 / 2} .
\end{aligned}
$$

It has been demonstrated (see, Sec. 2.3 of Ichikawa, Osaki (1992)) that the equations of mass and angular momentum conservation of the disk are written in our finite-difference scheme as

$$
\begin{aligned}
M_{\mathrm{disk}}^{\mathrm{new}}-M_{\mathrm{disk}} & =\left(\dot{M}_{\mathrm{tr}}-\dot{M}_{0}\right) d t \\
J_{\mathrm{disk}}^{\text {new }}-J_{\mathrm{disk}} & =\left(h_{\mathrm{LS}} \dot{M}_{\mathrm{tr}}-h_{0} \dot{M}_{0}-D_{\mathrm{total}}\right) d t,
\end{aligned}
$$

where $\dot{M}_{\mathrm{tr}}$ is the total mass transfer rate to the disk from the secondary star and it is given by

$\dot{M}_{\mathrm{tr}}=\int_{r_{0}}^{r_{N}} s(r) d r$

Here, $D_{\text {total }}$ is the total tidal torque exerted on the accretion disk, and expressed as follows:

$D_{\text {total }}=\int_{r_{0}}^{r_{N}} D d r$.

Equation (A7) means that the variation of the total disk mass is determined by the mass supply rate from the secondary minus the mass accretion rate onto the white dwarf, while the variation in the total angular momentum of the disk is determined by the angular momentum supply via the gas stream minus the angular momentum loss carried with the accreted matter minus the tidal removal of angular momentum from the disk (equation (A8)). That is, the exact conservation of mass and angular momentum in the disk is preserved during the time evolution in our numerical scheme.

The calculations at each time step are proceeded as follows. The necessary variables at the old time step are $N, r_{N}$, $\Sigma_{i-1 / 2}$, and $T_{\mathrm{c}, i-1 / 2}(i=1,2, \ldots, N)$. At first, we calculate $\dot{M}_{i}(i=0,1,2, \ldots, N-1)$ from the variables such as $W$ and $D$ by equation (A2). Next we compute $\Delta M_{i-1 / 2}^{\text {new }}$ by equation (A1), and derive $r_{N}$ at a new time step from equation (15) in Ichikawa, Osaki (1992). Finally we calculate $\Sigma_{i-1 / 2}^{\text {new }}$ by using $r_{N}$ at a new time step, and compute $T_{\mathrm{c}, i-1 / 2}^{\text {new }}$ from equation (A3).

We determine each time step $\Delta t$ to satisfy the following conditions in all of the meshes.

$$
\begin{array}{r}
\Delta t \leq \min \left(\frac{0.3(\Delta r)^{2}}{\nu}, \frac{0.3 \Delta r(2 \pi r \Sigma)}{|\dot{M}|}\right), \\
\left|T_{\mathrm{c}, \text { new }}-T_{\mathrm{c}}\right| \leq 0.03 T, \\
\left|\Sigma_{\text {new }}-\Sigma\right| \leq 0.03 \Sigma .
\end{array}
$$

The first one is the Courant condition to secure the numerical stability and means that each time step should be smaller than the diffusion timescale. Here, $T_{\mathrm{c} \text {,new }}$ and $\Sigma_{\text {new }}$ are the temperature at the mid-plane of the disk and the surface density after a new time step, respectively.

In our calculations in this paper, we set the first 90 annulus have the same width in logarithmic scales, and that the others do in linear scales.

For $r \geq 0.1 a(i \geq 111)$,

$d r=\frac{r_{\text {tidal }}-0.1 a}{90}$,

and for $r_{\text {input,min }} \leq r<0.1 a(91 \leq i \leq 110)$, 
$d r=\frac{0.1 a-r_{\text {input }, \min }}{20}$,

and for $r<r_{\text {input,min }}(i \leq 90)$,

$\log \left(r_{i}\right)-\log \left(r_{i-1}\right)=\frac{\log \left(r_{\text {input }, \text { min }}\right)-\log \left(r_{0}\right)}{90}$.

The reason why we divide the region where $r_{\text {input,min }} \leq r<$ $0.1 a$ into finer meshes than those in the nearby region is to smooth mass input in this region (see also Sec. 6.3).

\section{Appendix. 2. Frequency variations of the nodal precession rate}

We have calculated the variations of the nodal precession rate of a tilted rigid disk, and show them in the bottom panels of Figures 10 and 12. The precession rate normalized by the orbital frequency is estimated by the following equation (Papaloizou, Terquem 1995; Larwood 1998):

$\left|\epsilon_{-}^{*}\right|=\left|\nu_{\mathrm{nPR}} / \nu_{\mathrm{orb}}\right|=\frac{3}{4} \frac{G M_{2}}{a^{3}} \frac{\int \Sigma r^{3} d r}{\int \Sigma \Omega r^{3} d r} \cos \theta$,

where $\nu_{\mathrm{nPR}}$ is the nodal precession rate of a tilted disk and $\nu_{\mathrm{orb}}$ is the orbital frequency, respectively. Here, we set $\theta$ to be 0 . Also, $\epsilon_{-}^{*}$ is always negative, which means the retrograde precession. We also calculate $\left|\epsilon_{-}^{*}\right|$ depending only on the disk radius by assuming $\Sigma(r) \propto r^{-3 / 4}$, which comes from the standard disk (Shakura, Sunyaev 1973), via the following equation introduced in Osaki, Kato (2013):

$\left|\epsilon_{-}^{*}\right|=\left|\nu_{\mathrm{nPR}} / \nu_{\mathrm{orb}}\right|=\eta \frac{3}{7} \frac{q}{\sqrt{1+q}}\left(r_{N} / a\right)^{3 / 2} \cos \theta$,

where $\eta$ is a correction factor, which typically ranges from 0.8 to 1.2 (see Appendix 1 in Osaki, Kato 2013 for details). The estimated values by equation (A17) are displayed as solid lines and those by equation (A18) are given as dashed lines in the bottom panels of Figures 10 and 12 .

We first explain the variations in Model N1 (see the bottom panel of Figure 10). We see the precession rate estimated by equation (A17) (the solid line) well traces the expansion/contraction of the disk radius (the dashed line) in the short outbursts, but that it does not in the long outbursts, because $\Sigma(r)$ is drastically altered. At the onset of long outbursts, the whole disk enters the hot state and a large amount of mass accumulated at the outer disk is transported to the inner disk soon after the disk-radius expansion. The disk stays in the hot state for a while, and finally the cooling wave develops from the outer edge. It propagates inwards, and simultaneously sweeps up disk matter outward across the cooling front. Thus the complex changes in the nodal precession rate, i.e., the consecutive sets of the rapid increase and decrease and then again increase, reflect the three phenomena superimposed: the disk-radius expansion, the inward transport of a large amount of mass, and the redistribution of the disk mass by the cooling wave.

We next explain the variations of the nodal precession in
Model B1 (see the bottom panel of Figure 12). They show cyclic variations, and large and rapid variations occur around the large outburst. This kind of violent variations are very similar to those in the long outbursts in Model N1, but there exists one difference between these two cases, that is, the delay of the expansion of the disk to the increase of the nodal precession rate at the onset of the large outburst (see also the bottom panel of Figure 10). This is because the outward propagation of the heating wave precedes the disk-radius expansion in insideout outbursts, i.e., the mass redistribution by the heating wave occurs before the disk expansion, while in long outside-in outbursts in the case of non-tilted disks, the heating-wave propagation from the outer edge occurs almost simultaneously with the disk-radius expansion. Interestingly, the changes in the surfacedensity distribution near the outer edge of the disk govern the nodal precession rate rather than the disk-radius variations. On the other hand, the precession rate decreases during small outbursts in parallel with the disk-radius variations, unless the heating wave reaches the outermost region.

\section{Appendix. 3. Brief explanations of the results in models of the lowest and the highest test mass transfer rates}

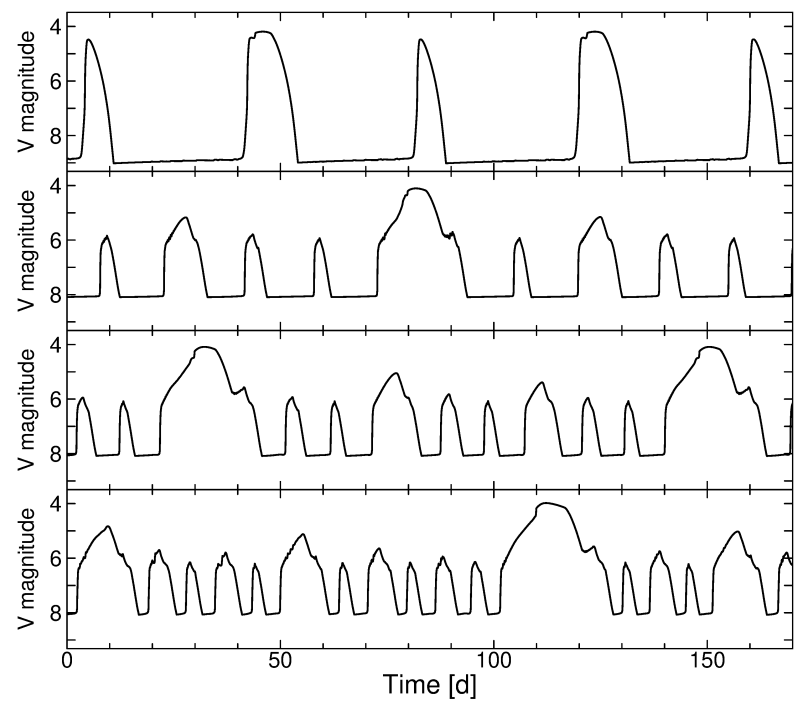

Fig. 17. Same as Figure 11 but for $\dot{M}_{\mathrm{tr}}=10^{16.5} \mathrm{~g} \mathrm{~s}^{-1}$ (Models N3, A3, $\mathrm{B} 3$, and $\mathrm{C} 3$ ).

In the case of $\dot{M}_{\mathrm{tr}}=10^{16.5} \mathrm{~g} \mathrm{~s}^{-1}$, the light curves alternate between several small outbursts and a large outburst even in Model C3 just as in Model A1 when the disk is tilted. This is because the cyclic accretion explained in Sec. 5.3 also occurs and because the mass supply rate in the inner disk is not enough to keep it hot even in the highly-tilted disk. In the case of $\dot{M}_{\mathrm{tr}}=10^{17.25}$, the disk in the standard non-tilted case set- 


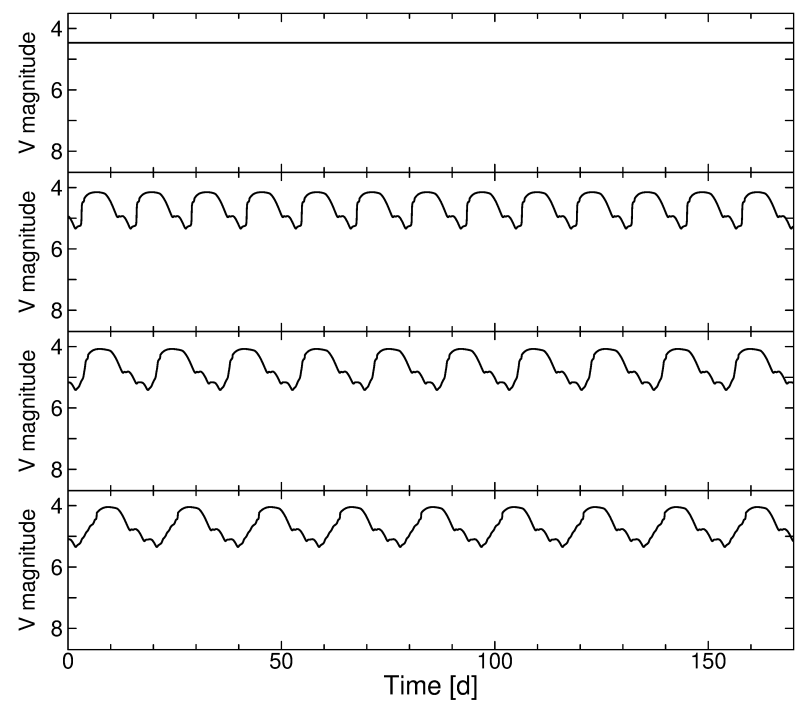

Fig. 18. Same as Figure 11 but for $\dot{M}_{\mathrm{tr}}=10^{17.25} \mathrm{~g} \mathrm{~s}^{-1}$ (Models N4, A4, $\mathrm{B} 4$, and $\mathrm{C} 4)$.

tles down to the hot steady disk, corresponding to NL stars. In tilted disks, the reduced mass supply in the outer edge cannot keep the outer disk hot continuously. The outer disk thus occasionally drops from the hot steady state, and it produces a light curve with small-amplitude variations.

\section{Appendix. 4. Details for test simulations with another set of binary parameters}

We have confirmed that the main results of our simulations do not depend on the binary parameters very much by testing with another set of binary parameters in Sec. 5.6. Here we present the binary parameters and meshes that we have applied to those simulations.

We have used the binary parameters of KIC 9406652, an IW And-type star. According to Gies et al. (2013), the orbital pe$\operatorname{riod}\left(P_{\text {orb }}\right)$ is $0.2545 \mathrm{~d}$, the white-dwarf mass $\left(M_{1}\right)$ is $0.9 M_{\odot}$, the mass of the secondary $\left(M_{2}\right)$ is $0.75 M_{\odot}$, the binary separation $(a)$ is $1.39 \times 10^{11} \mathrm{~cm}$, the tidal truncation radius $\left(r_{\text {tidal }}\right)$ is $0.328 a$, and the Lubow-Shu radius $\left(r_{\mathrm{LS}}\right)$ is $0.093 a$, respectively. The inner edge of the disk $\left(r_{0}\right)$ is the same as that in our previous calculations. We also have changed the number of the meshes from 200 to 190, and have given 80, 30, and 80 meshes in the three regions defined by equations (A14), (A15), and (A16) in Appendix 1. Then $r_{\text {input,min }}$ is estimated to be $0.053 a$ (Lubow, Shu 1975).

\section{References}

Anderson, N. 1988, ApJ, 325, 266

Armstrong, E., et al. 2013, MNRAS, 435, 707

Cannizzo, J. K. 1993, ApJ, 419, 318
Cannizzo, J. K., Shafter, A. W., \& Wheeler, J. C. 1988, ApJ, 333, 227

Cannizzo, J. K., \& Wheeler, J. C. 1984, ApJS, 55, 367

Cieslinski, D., Steiner, J. E., \& Jablonski, F. J. 1998, A\&AS, 131, 119

Cox, Arthur N., \& Stewart, John N. 1969, Nauchnye Informatsii, 15, 1

Davis, A. B., Shappee, B. J., Archer Shappee, B., \& ASAS-SN 2015,

American Astron. Soc. Meeting Abstracts, 225, \#344.02

Dubus, Guillaume, Otulakowska-Hypka, Magdalena, \& Lasota, JeanPierre 2018, A\&A, 617, A26

Echevarría, J., \& Michel, R. 2007, Rev. Mexicana Astron. Astrof., 43, 291

Fender, R., \& Belloni, T. 2004, ARA\&A, 42, 317

Flannery, B. P. 1975, MNRAS, 170, 325

Gies, D. R., et al. 2013, ApJ, 775, 64

Hōshi, R. 1979, 61, 1307

Hameury, J.-M., \& Lasota, J.-P. 2014, A\&A, 569, A48

Hameury, J.-M., Lasota, J.-P., \& Warner, B. 2000, A\&A, 353, 244

Hameury, J.-M., Menou, K., Dubus, G., Lasota, J.-P., \& Hure, J.-M. 1998, MNRAS, 298, 1048

Harvey, D., Skillman, D. R., Patterson, J., \& Ringwald, F. A. 1995, PASP, 107,551

Honeycutt, R. K. 2001, PASP, 113, 473

Ichikawa, S., \& Osaki, Y. 1992, PASJ, 44, 15

Ichikawa, S., \& Osaki, Y. 1994, PASJ, 46, 621

Kato, T. 2019, PASJ, 71, 20

Kato, T., et al. 2019, PASJ, accepted, arXiv:1911.01587

Larwood, J. 1998, MNRAS, 299, L32

Lubow, S. H., \& Shu, F. H. 1975, ApJ, 198, 383

McGowan, K. E., Priedhorsky, W. C., \& Trudolyubov, S. P. 2004, ApJ, 601,1100

Meyer, F. 1984, A\&A, 131, 303

Meyer, F., \& Meyer-Hofmeister, E. 1981, A\&A, 104, L10

Meyer, F., \& Meyer-Hofmeister, E. 1983, A\&A, 121, 29

Mineshige, S. 1986, PASJ, 38, 831

Mineshige, S., \& Osaki, Y. 1983, PASJ, 35, 377

Mineshige, S., \& Osaki, Y. 1985, PASJ, 37, 1

Mineshige, S., \& Wood, J. H. 1989, MNRAS, 241, 259

Montgomery, M. M., \& Martin, E. L. 2010, ApJ, 722, 989

Murray, J. R., Chakrabarty, D., Wynn, G. A., \& Kramer, L. 2002, MNRAS, 335, 247

Neilsen, J., Remillard, R. A., \& Lee, J. C. 2011, ApJ, 737, 69

Osaki, Y. 1996, PASP, 108, 39

Osaki, Y., \& Kato, T. 2013, PASJ, 65, 95

Paczyński, B. 1969, Acta Astron., 19, 1

Papaloizou, John C. B., \& Terquem, Caroline 1995, MNRAS, 274, 987

Ramsay, G., Hakala, P., Wood, M. A., Howell, S. B., Smale, A., Still, M., \& Barclay, T. 2016, MNRAS, 455, 2772

Ringwald, F. A. 1994, MNRAS, 270, 804

Rodríguez-Gil, P., et al. 2007, MNRAS, 377, 1747

Ross, Johnathan, \& Latter, Henrik N. 2017, MNRAS, 470, 34

Schlegel, E. M., \& Honeycutt, R. K. 2019, ApJ, 876, 152

Shakura, N. I., \& Sunyaev, R. A. 1973, A\&A, 24, 337

Simonsen, M. 2011, J. American Assoc. Variable Star Obs., 39, 66

Smak, J. 1983, ApJ, 272, 234

Smak, J. 1984, Acta Astron., 34, 93

Smak, J. 1984, Acta Astron., 34, 161

Szkody, P., et al. 2013, PASP, 125, 1421

Thompson, Susan E., et al. 2012, ApJ, 753, 86

Thorstensen, J. R., Fenton, W. H., \& Taylor, C. J. 2004, PASP, 116, 300

Warner, B. 1995, Cataclysmic Variable Stars (Cambridge: Cambridge 
University Press)

Watarai, Ken-ya, \& Mineshige, Shin 2003, ApJ, 596, 421

Wood, M. A., \& Burke, C. J. 2007, ApJ, 661, 1042

Wood, M. A., Montgomery, M. M., \& Simpson, J. C. 2000, ApJ, 535, L39 